\title{
CO excitation of normal star-forming galaxies out to $z=1.5$ as regulated by the properties of their interstellar medium
}

\author{
E. Daddi ${ }^{1}$, H. Dannerbauer ${ }^{2}$, D. Liu ${ }^{1,3,4}$, M. Aravena ${ }^{5}$, F. Bournaud ${ }^{1}$, F. Walter ${ }^{6}$, D. Riechers ${ }^{7}$, G. Magdis ${ }^{8,15}$,

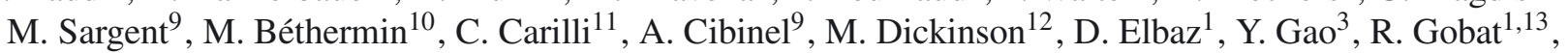 \\ J. Hodge ${ }^{11}$, and M. Krips ${ }^{14}$ \\ ${ }^{1}$ CEA Saclay, Laboratoire AIM-CNRS-Université Paris Diderot, Irfu/SAp, Orme des Merisiers, 91191 Gif-sur-Yvette, France \\ e-mail: edaddi@cea.fr \\ 2 Universität Wien, Institut für Astrophysik, Türkenschanzstrasse 17, 1180 Vienna, Austria \\ 3 Purple Mountain Observatory \& Key Laboratory for Radio Astronomy, Chinese Academy of Sciences, 210008 Nanjing, PR China \\ ${ }^{4}$ Graduate University of the Chinese Academy of Sciences, 19A Yuquan Road, Shijingshan District, 10049 Beijing, PR China \\ 5 Núcleo de Astronomía, Facultad de Ingeniería, Universidad Diego Portales, Av. Ejército Libertador 441, Santiago, Chile \\ ${ }^{6}$ Max-Planck Institute for Astronomy, Königstuhl 17, 69117 Heidelberg, Germany \\ 7 Department of Astronomy, Cornell University, 220 Space Sciences Building, Ithaca, NY 14853, USA \\ 8 Department of Physics, University of Oxford, Keble Road, Oxford OX1 3RH, UK \\ 9 Astronomy Center, Department of Physics and Astronomy, University of Sussex, Brighton BN1 9QH, UK \\ ${ }^{10}$ European Southern Observatory, Karl-Schwarzschild-Str. 2, 85748 Garching, Germany \\ 11 National Radio Astronomy Observatory, PO Box O, Socorro, NM 87801, USA \\ 12 National Optical Astronomical Observatory, 950 North Cherry Avenue, Tucson, AZ 85719, USA \\ 13 School of Physics, Korea Institute for Advanced Study, Heogiro 85, 130-722 Seoul, Republic of Korea \\ 14 IRAM - Institut de RadioAstronomie Millimétrique, 300 rue de la Piscine, 38406 Saint-Martin-d'Hères, France \\ 15 Institute for Astronomy, Astrophysics, Space Applications and Remote Sensing, National Observatory of Athens, 15236 Athens, \\ Greece
}

Received 23 September 2014 / Accepted 17 January 2015

\begin{abstract}
We investigate the $\mathrm{CO}$ excitation of normal (near-IR selected BzK) star-forming (SF) disk galaxies at $z=1.5$ using IRAM Plateau de Bure observations of the $\mathrm{CO}[2-1], \mathrm{CO}[3-2]$, and $\mathrm{CO}[5-4]$ transitions for four galaxies, including VLA observations of $\mathrm{CO}[1-0]$ for three of them, with the aim of constraining the average state of $\mathrm{H}_{2}$ gas. By exploiting previous knowledge of the velocity range, spatial extent, and size of the $\mathrm{CO}$ emission, we measure reliable line fluxes with a signal-to-noise ratio $>4-7$ for individual transitions. While the average CO spectral line energy distribution (SLED) has a subthermal excitation similar to the Milky Way (MW) up to CO[3-2], we show that the average $\mathrm{CO}[5-4]$ emission is four times stronger than assuming MW excitation. This demonstrates that there is an additional component of more excited, denser, and possibly warmer molecular gas. The ratio of CO[5-4] to lower- $J$ CO emission is lower than in local (ultra-)luminous infrared galaxies (ULIRGs) and high-redshift starbursting submillimeter galaxies, however, and appears to be closely correlated with the average intensity of the radiation field $\langle U\rangle$ and with the star formation surface density, but not with the star formation efficiency. The luminosity of the $\mathrm{CO}[5-4]$ transition is found to be linearly correlated with the bolometric infrared luminosity over four orders of magnitudes. For this transition, $z=1.5 \mathrm{BzK}$ galaxies follow the same linear trend as local spirals and (U)LIRGs and high-redshift star-bursting submillimeter galaxies. The CO[5-4] luminosity is thus empirically related to the dense gas and might be a more convenient way to probe it than standard high-density tracers that are much fainter than CO. We see excitation variations among our sample galaxies that can be linked to their evolutionary state and clumpiness in optical rest-frame images. In one galaxy we see spatially resolved excitation variations, where the more highly excited part of the galaxy corresponds to the location of massive SF clumps. This provides support to models that suggest that giant clumps are the main source of the high-excitation $\mathrm{CO}$ emission in high-redshift disk-like galaxies.
\end{abstract}

Key words. galaxies: evolution - galaxies: high-redshift - galaxies: starburst - galaxies: star formation - submillimeter: galaxies

\section{Introduction}

The nature of star formation in high-redshift galaxies and the physical properties of the interstellar medium (ISM) in which it is taking place are not very well understood as yet. Empirically, we now know that most star-forming (SF) galaxies have higher specific SF rates (sSFR) at higher redshifts, up to redshift 3 and possibly beyond (see, e.g., Sargent et al. 2014; and Madau \& Dickinson 2014, for recent compilations). This rise of sSFR appears to take place in a rather well-ordered way.
The increase is roughly independent of stellar mass at least for massive galaxies in the range $9.5<\log M / M_{\odot}<11.5$. This is the so-called main-sequence (MS) paradigm, in which starforming galaxies define a tight correlation between their stellar mass and SFR with a dispersion typically of a factor of two (Noeske et al. 2007; Elbaz et al. 2007, 2011; Daddi et al. 2007, 2009a; Pannella et al. 2009; Karim et al. 2011; Whitaker et al. 2012, 2014; Magdis et al. 2010; Rodighiero et al. 2011, 2014; Schreiber et al. 2015). When studying the most luminous systems (e.g., selected by means of their ultra-high infrared 
bolometric luminosity $-L_{\mathrm{IR}}(8-1000 \mu \mathrm{m})$, beyond $10^{12} L_{\odot}$ at $z=0$ or beyond $10^{13} L_{\odot}$ at $z=2$; Sargent et al. 2012), a rarer kind of galaxy with more extreme sSFR is most often found that likely is in a short-lived, possibly merging-driven, starburst phase. These objects are generally outliers to the MS (where, instead, disk-like systems typically live; Wuyts et al. 2011; Salmi et al. 2012; Forster-Schreiber et al. 2009) and account for a modest fraction of the global SFR density (e.g., 10-20\%; Rodighiero et al. 2011; Sargent et al. 2012; Hopkins et al. 2011). It is important to distinguish conceptually between these two kinds of galaxies (i.e., between MS and SB galaxies) to achieve a global understanding of star-forming galaxies through cosmic time.

When we concentrate on MS galaxies (or, almost equivalently, studying near-IR or optically selected galaxy samples), we now begin to obtain a fairly detailed empirical picture of the redshift evolution of some of their key physical properties, which change simultaneously to the rise of their sSFRs. First, their gas fractions appear to be rising substantially at least from $z=0$ to 2 , which begins from about $5-10 \%$ at $z=0$ to $40-50 \%$ at $z=2$ for stellar masses of about a few $10^{10} M_{\odot}$. This result has been obtained both from investigating $\mathrm{CO}$ emission lines mainly from the IRAM Plateau de Bure interferometer (PdBI, Daddi et al. 2008, 2010a, D10 hereafter; Tacconi et al. 2010, 2013; Geach et al. 2011) and was confirmed by gas-mass estimates obtained by converting dust masses (Magdis et al. 2011, 2012; Santini et al. 2014; Scoville et al. 2014; Magnelli et al. 2013; Genzel et al. 2015). Their ISM properties change as well: most notably, their dust temperatures increase, which is reflected by the increase with redshift of the average intensity of the radiation field $\langle U\rangle$ (Magdis et al. 2012; Magnelli et al. 2013; Béthermin et al. 2015; Genzel et al. 2015). Their metal content decreases, with the metallicity at fixed stellar mass declining by more than a factor of 2 toward $z=2$ (Erb et al. 2006; Maier et al. 2014; Steidel et al. 2014), and with some indications of a much more rapid decrease at higher redshifts (Troncoso et al. 2014). On the other hand, the ISM dust attenuation properties do not change much with only a modest rise of the attenuation at fixed stellar mass (e.g., Pannella et al. 2014; Burgarella et al. 2013). This is justified by the increase of the ratio of the dust to stellar mass (Tan et al. 2014). However, the difference between the attenuation of the HII regions and the stellar continuum appears to be smaller than in the local Universe (Kashino et al. 2013; Price et al. 2014; Pannella et al. 2014). Finally, MS galaxies from $z=0$ to beyond 2 appear to define a nonlinear trend in the SFR- $M_{\text {gas }}$ plane (either for integrated or specific quantities in the SchmidtKennicutt relation; KS; Daddi et al. 2010b; Genzel et al. 2010; Sargent et al. 2014), so that high-redshift galaxies, which have a higher SFR, also have higher star formation efficiencies (SFE = $\left.S F R / M_{\text {gas }}\right)$. This wealth of empirical information still does not suffice to clarify the physical properties of the ISM. Studies of emission-line ratios suggest that the properties of the ionizing radiation field are different at $z=2$, with harder and possibly more intense spectra (Steidel et al. 2014) that qualitatively agree with the observations of higher $\langle U\rangle$ (dust is a bolometric tracer and thus cannot distinguish harder from more intense fields). This might affect the kinetic temperature of the gas. At the same time, the gas densities might be changing, given the higher gas fractions and possibly decreasing galaxy sizes toward higher redshifts (e.g., van der Wel et al. 2014).

One important piece of information to gain further insight into the ISM properties is the study of the excitation of the $\mathrm{CO}$ emission, that is, the relative luminosity ratio of $\mathrm{CO}$ lines with different rotational quantum number $\left(J_{\text {upper }}\right)$. CO is the most luminous tracer of molecular hydrogen $\left(\mathrm{H}_{2}\right)$, the fuel for star formation in galaxies. The specific intensity distribution of high- $J$ versus low- $J \mathrm{CO}$ is sensitive to the $\mathrm{H}_{2}$ gas density and temperature, albeit in a somewhat degenerate way, providing insights into the physical properties of the gas. The effective critical densities for excitation rise from $\sim 300 \mathrm{~cm}^{-3}$ for $\mathrm{CO}[1-0]$, which is thus most sensitive to the total gas reservoir, including more diffuse components, to $\sim 10^{4} \mathrm{~cm}^{-3}$ for CO[5-4], for example, so that $J>1$ transitions are increasingly sensitive to the denser star-forming gas (e.g., Solomon \& van den Bout 2005). On the other hand, studying $\mathrm{CO}$ excitation properties of high-redshift MS galaxies is interesting empirically as well, as a means to further understand their nature and the possible role of mergers or starbursts in driving their properties. It is well known locally that empirically and observationally, different $\mathrm{CO}$ excitation properties characterize spiral galaxies as opposed to merging-driven (ultra-) luminous infrared galaxies ((U)LIRGs). The latter are much more highly excited in their high- $J$ CO transitions (Weiss et al. 2007; Papadopoulos et al. 2012, P12 hereafter). However, CO spectral line energy distributions (SLEDs) by themselves are not necessarily good indicators of the nature of star formation and galaxy types. Regardless of whether they are vigorously SF disks or mergers, CO SLEDs of warm gas will be similar. It is primarily the fraction of dense to total gas that can indicate a SF mode (merger vs. disk; e.g., Daddi et al. 2010b; Zhang et al. 2014), if it can be constrained by the available SLEDs. Not much is observationally known about the $\mathrm{CO}$ excitation properties of indisputably normal MS galaxies at high redshift. The most detailed information is available for IR-luminous systems selected either as submillimeter galaxies (SMGs; e.g., Bothwell et al. 2013; Riechers et al. 2013) or strong lenses (Danielson et al. 2011; Cox et al. 2011; Combes et al. 2012; Scott et al. 2011; Spilker et al. 2014). In a pilot study, Dannerbauer et al. (2009) presented $\mathrm{CO}$ [3-2] observations of one $z=1.5$ galaxy (BzK-21000, further discussed in this paper) that was previously detected in $\mathrm{CO}[2-1]$, finding a low $\mathrm{CO}[3-2] / \mathrm{CO}[2-1]$ ratio fairly consistent with the Milky Way (MW). Aravena et al. (2014) presented CO[1-0] observations made with the Karl G. Jansky Very Large Array (VLA) of three BzK-selected and one UV-selected galaxies which, compared to $\mathrm{CO}$ [2-1] or $\mathrm{CO}$ [3-2] observations of the same objects, display fairly low excitation ratios. Not much more is known about the excitation properties of MS-selected galaxies for higher $J$ transitions, that is, those most sensitive to warm and dense gas. We aim to begin to remedy this with the present study with new $\mathrm{CO}$ [5-4] observations. This is particularly important as the study of SLEDs up to $\mathrm{CO}$ [3-2] alone can be fairly degenerate in clarifying the type of underlying ISM conditions (e.g., Dannerbauer et al. 2009; Papadopoulos et al. 2014).

There are empirical reasons to expect evolution in the CO spectral energy distributions (SEDs) of MS disk-like galaxies at high redshift compared to local spirals and the MW, mostly because of the previously discussed evolution in the related physical properties. For example, the rise in SFE suggests the parallel increase of the fraction of dense versus total molecular gas (e.g., Daddi et al. 2010b). The increase of the mean radiation field intensity $\langle U\rangle$ is probably directly reflected in rising gas temperatures. All of this would suggest the idea that CO SLEDs are not identical to the MW SLEDs at higher $J$-transition, where some higher level of gas excitation is probably present. This kind of qualitative assessment agrees with theoretical predictions. For example, P12 predicted stronger high- $J$ emission heated by turbulence and/or cosmic rays in these systems with higher SFEs than local spirals. Similarly, Narayanan \& Krumholz (2014) 
Table 1. Observation summary table.

\begin{tabular}{lcccccccc}
\hline \hline Source & $\begin{array}{c}\mathrm{RA}^{a} \\
\mathrm{~J} 2000\end{array}$ & $\begin{array}{c}\mathrm{Dec}^{a} \\
\mathrm{~J} 2000\end{array}$ & Transition & $\begin{array}{c}\text { Frequency } \\
\mathrm{GHz}\end{array}$ & Obs.dates & $\begin{array}{c}T_{\text {int }}^{b} \\
\mathrm{~h}\end{array}$ & $\begin{array}{c}\text { Combined beam }^{c} \\
\mathrm{rms} \\
\mu \mathrm{Jy}\end{array}$ \\
\hline BzK-4171 & $12: 36: 26.520$ & $62: 08: 35.48$ & $\mathrm{CO}[3-2]$ & 140.282 & $04 / 2009$ & 12.3 & $5.0^{\prime \prime} \times 3.2^{\prime \prime}, \mathrm{PA}=52^{\circ}$ & 290 \\
& & & $\mathrm{CO}[5-4]$ & 233.780 & $01-09 / 2011$ & 11.5 & $2.8^{\prime \prime} \times 2.0^{\prime \prime}, \mathrm{PA}=113^{\circ}$ & 460 \\
\hline BzK-16000 & $12: 36: 30.114$ & $62: 14: 28.29$ & $\mathrm{CO}[3-2]$ & 137.057 & $04 / 2009$ & 9.8 & $5.2^{\prime \prime} \times 3.5^{\prime \prime}, \mathrm{PA}=48^{\circ}$ & 410 \\
& & & $\mathrm{CO}[5-4]$ & 228.225 & $04-07 / 2011$ & 6.8 & $2.9^{\prime \prime} \times 2.2^{\prime \prime}, \mathrm{PA}=80^{\circ}$ & 390 \\
\hline BzK-17999 & $12: 37: 51.827$ & $62: 15: 19.90$ & $\mathrm{CO}[3-2]$ & 143.246 & $04-05 / 2009$ & 10.7 & $4.3^{\prime \prime} \times 3.4^{\prime \prime}, \mathrm{PA}=75^{\circ}$ & 370 \\
& & & $\mathrm{CO}[5-4]$ & 238.719 & $05-10 / 2011$ & 12.9 & $2.5^{\prime \prime} \times 2.1^{\prime \prime}, \mathrm{PA}=92^{\circ}$ & 310 \\
\hline BzK-21000 & $12: 37: 10.642$ & $62: 22: 34.35$ & $\mathrm{CO}[3-2]^{e}$ & 136.970 & $05-06 / 2008$ & 9.7 & $4.0^{\prime \prime} \times 3.1^{\prime \prime}, \mathrm{PA}=72^{\circ}$ & 260 \\
& & & $\mathrm{CO}[5-4]$ & 228.574 & $05-10 / 2009$ & 8.7 & $2.9^{\prime \prime} \times 2.0^{\prime \prime}, \mathrm{PA}=50^{\circ}$ & 490 \\
\hline
\end{tabular}

Notes. ${ }^{(a)}$ The coordinates correspond to the fixed positions for our CO extractions, as detailed in Sect. 3.1. ${ }^{(b)}$ Equivalent to the on-source integration time of six antennas. ${ }^{(c)}$ Beam resulting from combining all available configuration and imaging with natural weighting. These are the beams sizes displayed in Figs. 3 and 4. ${ }^{(d)}$ Noise per beam averaged over a spectral range corresponding to $400 \mathrm{~km} \mathrm{~s}^{-1}$. ${ }^{(e)}$ These data have previously been presented in Dannerbauer et al. (2009).

suggested that the CO SLED is driven by the SFR surface density $\left(\Sigma_{\mathrm{SFR}}\right)$, with galaxies with highest $\Sigma_{\mathrm{SFR}}$ having more excited high- $J$ components. More recently, fully detailed very high resolution numerical hydrodynamical simulations of high- $z$ disks have been used by Bournaud et al. (2015, B15 hereafter) to predict the evolution of $\mathrm{CO}$ excitation, confirming the expectation for a significant high-excitation component beyond $\mathrm{CO}$ [3-2]. Semi-analytic models also predict increasing $\mathrm{CO}$ excitation toward $z \sim 2$ (Popping et al. 2014; Lagos et al. 2012).

In this paper we present $\mathrm{CO}[5-4]$ and $\mathrm{CO}$ [3-2] observations of four BzK galaxies at $z=1.5$. Coupled with existing measurements at $\mathrm{CO}[2-1]$ and $\mathrm{CO}[1-0]$, they allow us to derive CO SLEDs for these objects extending to $\mathrm{CO}$ [5-4], study their excitation properties, and compare them with other physical parameters and with model predictions. The paper is organized as follows: in Sects. 2 and 3 we present the dataset and the measurements performed on our data. In Sect. 4 we discuss results on the CO SLEDs of the BzK galaxies in comparison with other galaxy populations at low and high redshift and with theoretical models. Section 5 discusses the physical constraints on the gas density and temperature that can be obtained from our data using large velocity gradient (LVG) modeling. We discuss our findings in Sect. 6, where we try to identify the main driver of CO excitation and find that the mean radiation field intensity $\langle U\rangle$ appears to be the best tracer together with $\Sigma_{\mathrm{SFR}}$. We also find that the BzK galaxies obey a linear correlation between the $\mathrm{CO}[5-4] \mathrm{lu}-$ minosity and $L_{\mathrm{IR}}$, which is also followed by other galaxy populations. Finally, we discuss the evidence that the $\mathrm{CO}$ excitation is linked to giant clumps. We use a Chabrier (2003) IMF when specifying SFRs and stellar masses, and adopt a standard WMAP cosmology.

\section{Observations}

The targets of our observations are four near-IR-selected galaxies with high signal-to-noise $(\mathrm{S} / \mathrm{N})$ detections of $\mathrm{CO}[2-1]$ taken from D10: BzK-4171, BzK-16000, BzK-17999, and BzK-21000, with redshifts spanning the range $1.414<z<$ 1.523 and SFRs placing them in the star-forming MS. There is no sign of AGN emission in these galaxies, from the two Ms Chandra data, radio $1.4 \mathrm{GHz}$ continuum, optical spectroscopy, Spitzer IRAC colors, IRS Spitzer spectroscopy in 4171 and 21000, and mid- to far-IR decomposition (D07; Pope et al. 2013; Daddi et al., in prep.). These galaxies have been observed in $\mathrm{CO}[2-1]$ in at least two different configurations with the IRAM PdBI, including an extended configuration (B and/or A) that revealed resolved SF activity with the resulting data, which allows constraining their $\mathrm{CO}[2-1]$ spatial sizes $(\mathrm{D} 10)^{1}$. The fairly accurate $\mathrm{CO}[2-1]$ fluxes available for these galaxies were an excellent starting point from which to investigate their $\mathrm{CO}$ excitation properties with the follow-up of different $\mathrm{CO}$ transitions.

\subsection{IRAM PdBI}

We observed our targets in the $\mathrm{CO}[3-2]$ (345.796 $\mathrm{GHz}$ rest frame, redshifted to the $2 \mathrm{~mm}$ band) and $\mathrm{CO}[5-4](576.267 \mathrm{GHz}$ rest frame, redshifted to the $1 \mathrm{~mm}$ band) transitions during several observing campaigns of the IRAM PdBI, spanning the Winter 2008 to Winter 2011 IRAM semesters (see Table 1). All observations were obtained in compact configurations given that we were mainly interested in the total fluxes for this detection experiment, resulting in synthesized beams of 4-5" for $\mathrm{CO}$ [3-2] and 2-3" for $\mathrm{CO}$ [5-4] (Table 1). All sources are expected to remain unresolved in the data at this resolution. The $\mathrm{CO}$ [3-2] observations of BzK-21000 were the first to be obtained, have been published in Dannerbauer et al. (2009), and are also used here. The $\mathrm{CO}$ [3-2] observations of all four sources and the $\mathrm{CO}$ [5-4] data of BzK-21000 were obtained with the previous-generation IRAM correlator, with a total bandwidth of $1 \mathrm{GHz}$, while for the other three galaxies the $\mathrm{CO}[5-4]$ observations performed since 2011 benefited from the new Widex correlator, which has a total bandwidth of $3.6 \mathrm{GHz}$. All data were taken in dual-polarization mode. The combined on-source time is $82 \mathrm{~h}$ of six-antenna equivalent time (part of the data were taken with only five antennas), corresponding to a total of about $130 \mathrm{~h}$ of PdBI observing time when considering overheads. The data were reduced and calibrated using the GILDAS software package CLIC. The final data, imaged using natural weighting, typically have noise in the range of $250-450 \mu \mathrm{Jy}$ integrated over $400 \mathrm{~km} \mathrm{~s}^{-1}$ bandwidth, which is representative of the line-widths of the target BzK galaxies. This corresponds to roughly $0.15 \mathrm{Jy} \mathrm{km} \mathrm{s}^{-1}$ when integrated over the velocity range. For two of the sources we slightly offset the pointing of the

\footnotetext{
1 The sample of D10 contains two more BzK sources that were only observed in one configuration and thus have lower $\mathrm{S} / \mathrm{N} \mathrm{CO}[2-1]$ detections.
} 
$\mathrm{CO}$ [3-2] observations to also gather information on interesting nearby sources (GN10 for BzK-16000; Daddi et al. 2009b; and the GN20/GN20.2ab sources close to BzK-21000; Daddi et al. 2009a; Carilli et al. 2010; Hodge et al. 2012, 2013, 2015; Tan et al. 2013, 2014). The sensitivity loss due to primary beam attenuation at off-center position was minimal, only $10 \%$ at the position of the BzK galaxies. We corrected all measurements for the flux loss. We expect that fluxes are affected by systematic calibration uncertainties at a level lower than $\pm 10 \%$, based on well-studied and frequently observed calibrators (MWC349 primarily) and on the monitored efficiency of PdBI antennas. This is below our measurement errors.

\subsection{VLA}

Three of our sources were observed in their $\mathrm{CO}[1-0]$ emission (115.271 GHz rest frame, redshifted to $\sim 6.6 \mathrm{~mm}$ ) at the VLA: BzK-4171, BzK-16000, and BzK-21000 (Aravena et al. 2010, 2014). No CO[1-0] data are available for BzK-17999. A first observational campaign was conducted with the VLA during 2009 in the $\mathrm{C}$ and $\mathrm{D}$ configurations using two $50 \mathrm{MHz}$ channels, with results published by Aravena et al. (2010). The same three targets were re-observed with the VLA during 2010-2011 using the WIDAR correlator, which covers the expected line frequencies with $1282 \mathrm{MHz}$ channels. The new VLA observations are described in detail in Hodge et al. (2012), and measurements for the BzK galaxies were published in Aravena et al. (2014). In this paper we use the combination of new and old VLA observations to extend the CO SLEDs to the CO[1-0] transitions, performing new flux measurements for consistency with the IRAM data. The resulting spatial resolutions were on the order of 1.5-2" (Aravena et al. 2010, 2014), except for BzK-21000, which benefited from part of the data being taken at a higher resolution. The VLA images of our targets are shown in Aravena et al. (2010, 2014).

\section{Measurements}

Interferometric data are effectively 3D cubes including spatial and velocity information. Measuring a $\mathrm{CO}$ flux involves integrating the data over a certain velocity range and then extracting the flux with assumptions on the spatial profile. Hence, the global error connected to absolute flux measurements can be substantially affected by the possible ambiguity in choosing the velocity range and spatial position and extent of the source. To build CO SLEDs as accurately as possible, we exploited the full set of information contained in the different $\mathrm{CO}$ transitions that we observed for each source.

\subsection{IRAM PdBI}

Consistently with the $\mathrm{CO}$ flux values reported in D10, all $\mathrm{CO}$ measurements reported in this paper were extracted using circular Gaussian models ${ }^{2}$ for the sources and fitting the visibilities in the $u v$ space using $u v f i t$ under MAPPING in GILDAS, with fixed CO sizes as given in the right panel of Fig. 4 of D10 (see also Table 2 here). These CO sizes are consistent, on average, with the optical sizes measured with the Hubble Space

\footnotetext{
2 A circular Gaussian model has only one additional parameter compared to a PSF model, i.e. the size FWHM, and hence is best suited for somewhat resolved measurements with limited $\mathrm{S} / \mathrm{N}$ as in our case.
}

Telescope (HST; D10). Given the lack of high spatial resolution configurations in the new observations of $\mathrm{CO}[3-2]$ and $\mathrm{CO}[5-4]$ (Table 1), we cannot improve on those size measurements, but at the same time, the measured fluxes of these high- $J$ transitions are rather insensitive to the exact Gaussian full width at half maximum used for extraction (which is always substantially smaller than the synthesized beam).

To define the CO positions and velocity widths for the high- $J$ $\mathrm{CO}$ observations, we proceeded iteratively. Starting from the IRAM observations of $\mathrm{CO}[2-1]$ from D10 that already have fairly high $\mathrm{S} / \mathrm{N}$ and thus fairly small positional errors, we collapsed the data cube using velocity ranges defined from $\mathrm{CO}[2-1]$ to obtain single channel datasets of $\mathrm{CO}$ [3-2] and $\mathrm{CO}$ [5-4] for each source. We fitted the $u v$ data with an appropriate circular Gaussian model with free spatial centering, determining the position and the error of the $\mathrm{CO}$ emission that we could compare to the $\mathrm{CO}[2-1]$ positions with their uncertainties. In all cases the high- $J \mathrm{CO}$ positions agreed with the old $\mathrm{CO}[2-1]$ ones, except for BzK-4171, in which a small offset was suggested. In that case we defined a new best $\mathrm{CO}$ position by combining the independent measurements in all transitions weighted with their noise, resulting in a small offset of $0.2^{\prime \prime}$ to the north ( $2 \sigma$ significance) with respect to the old $\mathrm{CO}[2-1]$ position, comparable to its $1 \sigma$ uncertainty (note that the $\mathrm{CO}$ contours in the top left panel of Fig. 2 in D10 were slightly offset to the south with respect to the HST imaging of the galaxy; this probably is an effect of noise that we now correct for). We then extracted $\mathrm{CO}$ spectra for all transitions using $25 \mathrm{~km} \mathrm{~s}^{-1}$ channels at fixed spatial positions. We produced averages of the $\mathrm{CO}[2-1], \mathrm{CO}[3-2]$, and $\mathrm{CO}[5-4]$ spectra using the global $\mathrm{S} / \mathrm{N}$ in each transition to determine its weight and inspected them to verify whether the velocity ranges defined in D10 were still the most accurate definition of the spectral windows. This was the case for all sources, except again for BzK-4171, where we reduced the velocity range by excluding two channels on the blue side and one on the red side, resulting in a $12 \%$ smaller spectral range of extraction because no sign of a signal was present at these edges when combining the three $\mathrm{CO}$ transitions. By reiterating the spatial-positioning check for BzK-4171 with the new channels, we verified that the procedure had converged.

We caution here that while this procedure allows us to exploit all available information to provide highest fidelity measurements of CO SLEDs within the chosen velocity range, which is determined by the $\mathrm{CO}[2-1]$ observations primarily and also by $\mathrm{CO}$ [3-2] and $\mathrm{CO}$ [5-4] consistently, we cannot rule out that some $\mathrm{CO}$ signal exists in broader components that extend beyond the velocity ranges where our measurements are obtained. Such broad components are sometime seen in starburst-like galaxies (Riechers et al. 2011) and appear to be more prominent in low- $J$ transitions, hence could alter the total CO SLEDs. Our SLEDs thus neglect such putative high-velocity wings if they are present in our targets.

The final CO spectra for all $J=2,3,5$ transitions for the four BzK galaxies are shown in Fig. 1, their weighted average is plotted in Fig. 2. We also produced images of our sources in the $\mathrm{CO}[3-2]$ and $\mathrm{CO}[5-4]$ transitions (Figs. 3 and 4) using a natural weighting scheme. Images and spectra were created after estimating (and subtracting) the continuum level, which is mostly relevant for $\mathrm{CO}$ [5-4], as described below (Sect. 3.2). All $\mathrm{CO}$ fluxes and their uncertainties are reported in Table 2 . We also computed and report in the table $\mathrm{CO}$ luminosity ratios, expressed as $R_{J 1} J 2=I_{\mathrm{CO}}[J 1 J 1-1] / I_{\mathrm{CO}}[J 2 J 2-1] \times(J 2 / J 1)^{2}$. These are equivalent to brightness temperature ratios between transition $J 1$ and $J 2$ only in the limit of small Rayleygh-Jeans corrections 

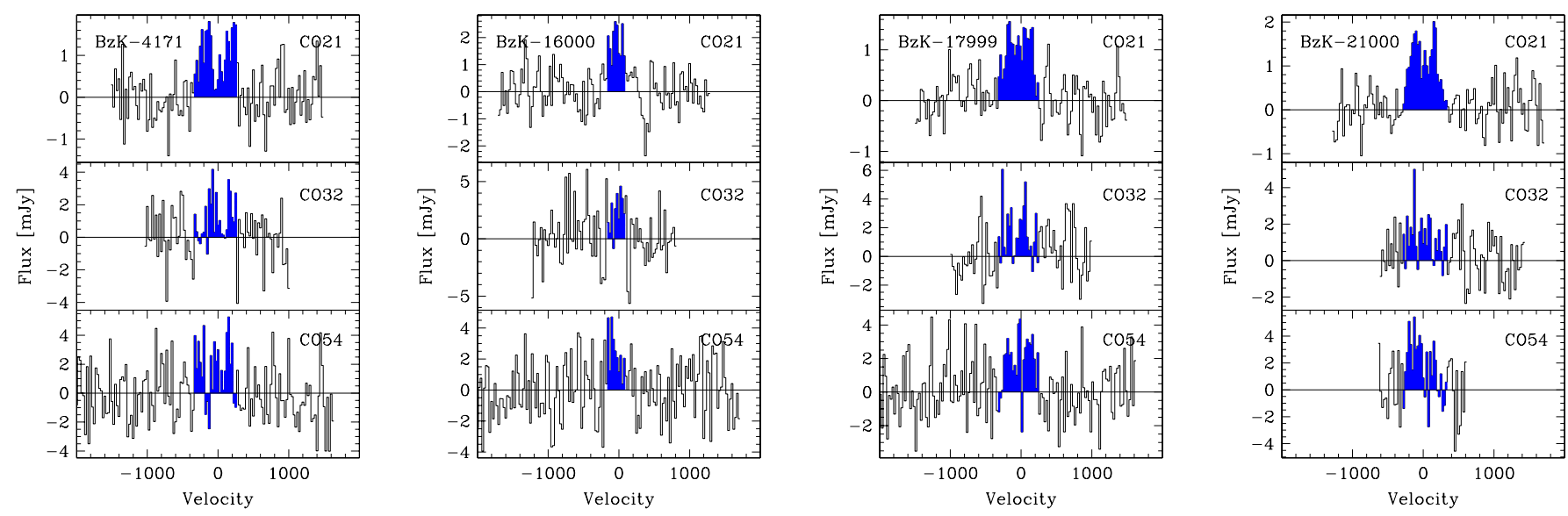

Fig. 1. Multi-transition spectra of $\mathrm{CO}[2-1]$ (top), $\mathrm{CO}$ [3-2] (middle) and $\mathrm{CO}[5-4]$ (bottom), for each galaxy in our sample extracted by fitting circular Gaussian models convolved with the beam at the position of the galaxies in steps of $25 \mathrm{~km} \mathrm{~s}^{-1}$. The CO[2-1] data are taken from D10, the CO[3-2] data for BzK-21000 from Dannerbauer et al. (2009). The shaded regions correspond to the velocity range (kept fixed for all transitions of a given galaxy), over which fluxes are measured. Continuum levels as measured in the data and constrained from our multiwavelength modeling have been subtracted. Zero velocity corresponds to the accurate $\mathrm{CO}[2-1]$ redshifts from $\mathrm{D} 10$.
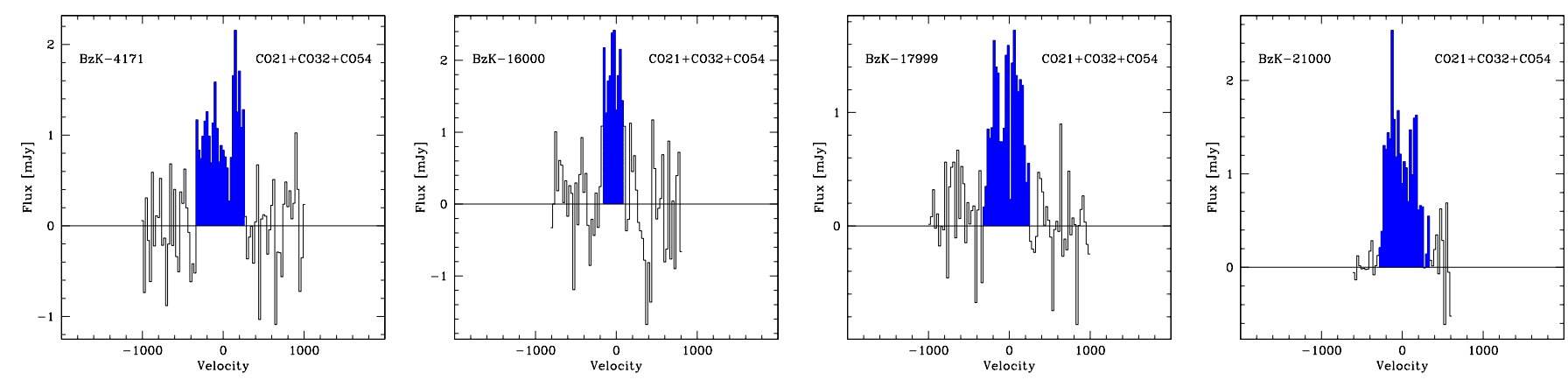

Fig. 2. $\mathrm{CO}$ spectra averaged over the $\mathrm{CO}[2-1], \mathrm{CO}[3-2]$, and $\mathrm{CO}[5-4]$ transitions for each galaxy, after continuum subtraction, weighted with the $\mathrm{S} / \mathrm{N}$ in each transition.

Table 2. CO flux measurements and luminosity ratios.

\begin{tabular}{lcccccccccc}
\hline \hline Source & Redshift $^{a}$ & $\begin{array}{c}\Delta v^{b} \\
\mathrm{~km} \mathrm{~s}^{-1}\end{array}$ & $\begin{array}{c}I_{\mathrm{CO}[1-0]} \\
\mathrm{Jy} \mathrm{km} \mathrm{s}^{-1}\end{array}$ & $\begin{array}{c}I_{\mathrm{CO}[2-1]} \\
\mathrm{Jy} \mathrm{km} \mathrm{s}^{-1}\end{array}$ & $\begin{array}{c}I_{\mathrm{CO}[3-2]} \\
\mathrm{Jy} \mathrm{km} \mathrm{s}^{-1}\end{array}$ & $\begin{array}{c}I_{\mathrm{CO}[5-4]} \\
\mathrm{Jy} \mathrm{km} \mathrm{s}^{-1}\end{array}$ & $\begin{array}{c}f_{1.3 \mathrm{~mm}^{d}} \\
\mathrm{mJy}\end{array}$ & $R_{21}$ & $R_{31}$ & $R_{51}$ \\
\hline 4171 & 1.4652 & 600 & $0.178 \pm 0.039$ & $0.65 \pm 0.08$ & $0.76 \pm 0.17$ & $1.20 \pm 0.23$ & $0.14 \pm 0.15$ & $0.92 \pm 0.23$ & $0.47 \pm 0.15$ & $0.27 \pm 0.08$ \\
16000 & 1.5250 & 250 & $0.240 \pm 0.027$ & $0.46 \pm 0.06$ & $0.58 \pm 0.14$ & $0.69 \pm 0.16$ & $0.44 \pm 0.14$ & $0.48 \pm 0.08$ & $0.27 \pm 0.07$ & $0.12 \pm 0.03$ \\
17999 & 1.4138 & 575 & - & $0.58 \pm 0.06$ & $0.97 \pm 0.18$ & $1.28 \pm 0.17$ & $0.30 \pm 0.11$ & & & \\
21000 & 1.5213 & 525 & $0.161 \pm 0.026$ & $0.66 \pm 0.07$ & $0.83 \pm 0.16$ & $1.49 \pm 0.32$ & $0.84 \pm 0.36$ & $1.02 \pm 0.20$ & $0.57 \pm 0.14$ & $0.36 \pm 0.10$ \\
Average $^{c}$ & - & - & $0.193 \pm 0.018$ & $0.59 \pm 0.04$ & $0.73 \pm 0.09$ & $1.12 \pm 0.14$ & - & $0.76 \pm 0.09$ & $0.42 \pm 0.07$ & $0.23 \pm 0.04$ \\
\hline
\end{tabular}

Notes. ${ }^{(a)}$ Redshifts are taken from D10. ${ }^{(b)}$ Velocity range for the flux measurements. ${ }^{(c)}$ Average of the three BzK galaxies with all four transition measurements (BzK-4171, 16000 and 21000). Uncertainties are standard errors of the mean. ${ }^{(d)}$ Continuum flux density at the rest frequency of $\mathrm{CO}[5-4]$ (the $\mathrm{CO}$ fluxes listed in this table were all corrected for the underlying continuum).

(see Sect. 2.6 in Genzel et al. 2010). The final extraction coordinates are listed in Table 1. All measurements of $\mathrm{CO}[3-2]$ and $\mathrm{CO}[5-4]$ result in at least a $S / N>4$, and half of them have a $S / N>5$. The noise structure of the data is not fully homogeneous, however, and the CO maps in some cases reveal nonGaussian departures corresponding to negative peaks. However, performing measurements over fixed velocity and spatial positions, largely determined from $\mathrm{CO}[2-1]$, can help to minimize the impact of these effects given the high significance of the detection of this line in each source, that is to say, we are not chasing high- $\sigma$ noise peaks in the data cubes. We also emphasize that even non-detections or lower $\mathrm{S} / \mathrm{N}$ measurements than presented here would have provided meaningful constraints on the higher excitation part of the SLED because our deep observations would comfortably have detect these transitions if they were thermally excited.

\subsection{VLA}

For the VLA data, which are the combination of datasets obtained with different correlators over the years, we did not fit the observations in $u v$ space to measure fluxes (because this is 

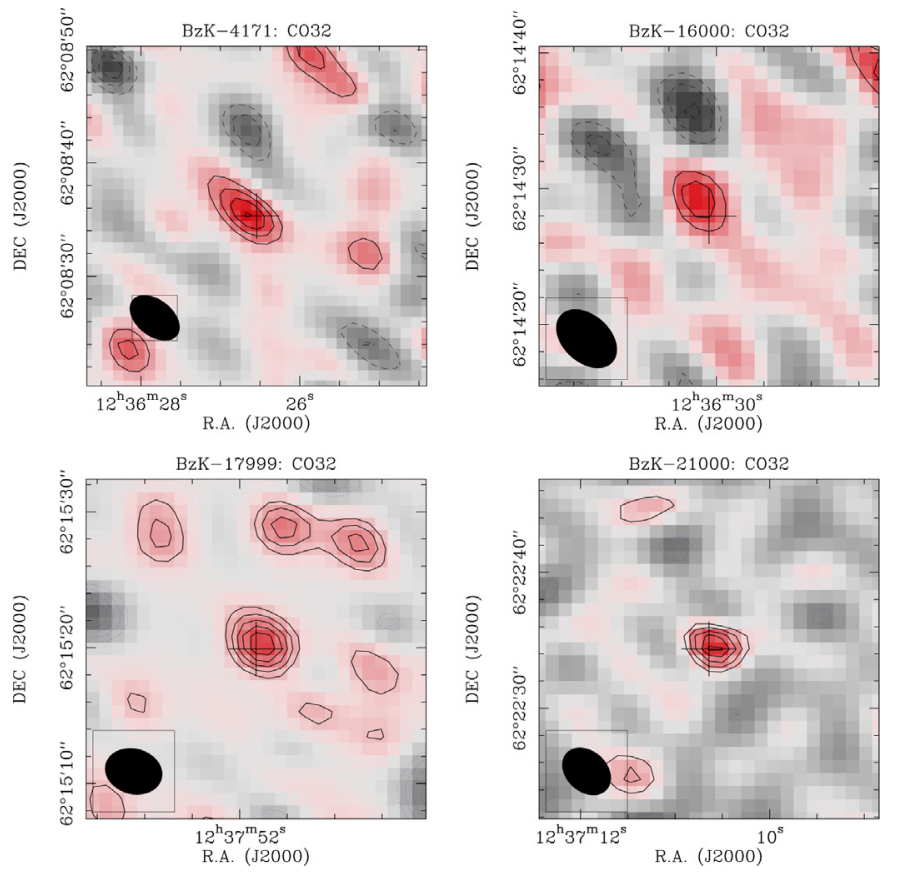

Fig. 3. Velocity-averaged maps of the CO[3-2] emission of our galaxies, using natural weighting. Contour levels start at $\pm 2 \sigma$ and increase in steps of $1 \sigma$. The size and orientation of the beam is indicated in the bottom left corner. The cross in each panel is centered on the $\mathrm{CO}$ positions from Table 1.
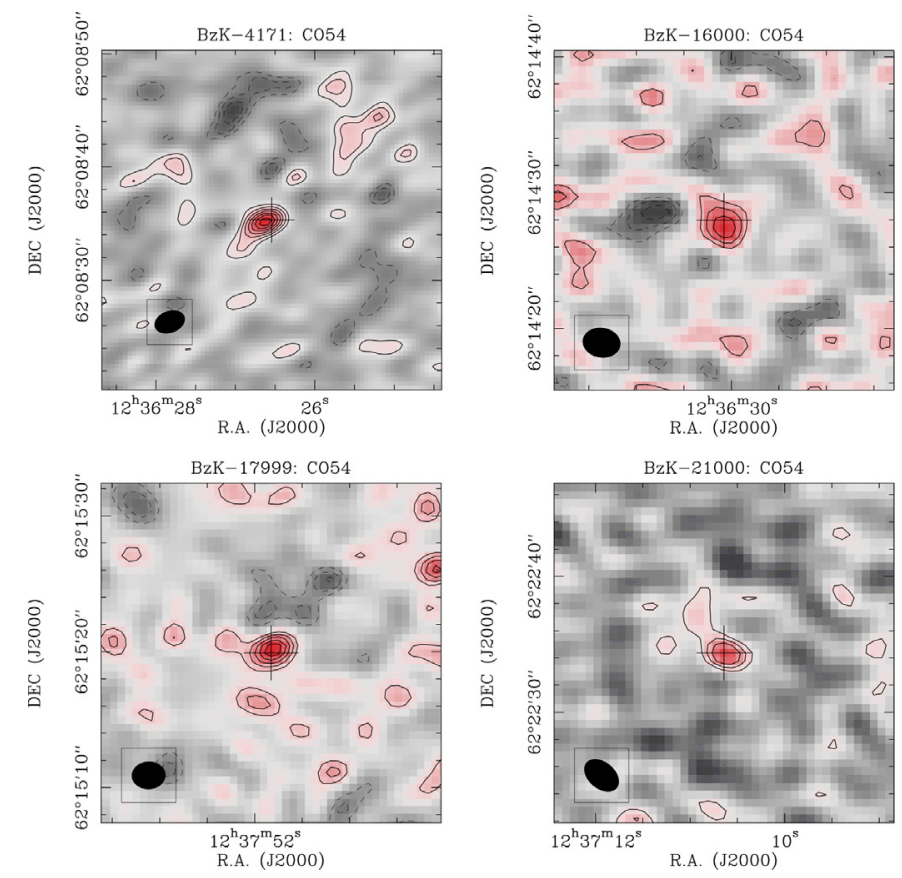

Fig. 4. Same as Fig. 3, but for the CO[5-4] observations. Continuum emission has been subtracted here, as discussed in the text.

too complex operationally) but produced images using a natural weighting scheme, based on the velocity information derived from IRAM, and performed flux measurements directly on the images. We were unable to exactly match the $25 \mathrm{~km} \mathrm{~s}^{-1}$ velocity channel sampling we used at IRAM (corresponding to $3.5-4 \mathrm{MHz}$ at $45 \mathrm{GHz}$ ) because of the poorer spectral resolution of the older VLA correlator ( $50 \mathrm{MHz}$ channels), but in all cases we were able to fully cover the velocity extent of the line, sometimes with bandwidths wider by up to $50 \mathrm{~km} \mathrm{~s}^{-1}$ (which could help alleviate problems with the possible broader $\mathrm{CO}[1-0]$ components discussed in the previous section).

We then proceeded to measure $\mathrm{CO}[1-0]$ fluxes in the resulting images by fitting the same circular Gaussian models as were used for higher- $J$ CO, but this time with galfit (Peng et al. 2010), and using the synthesized beam as the point spread function (PSF). Uncertainties were estimated by measuring the rms of the pixel values in the images, which is appropriate for the noise of point sources, and scaling these values up by the ratio of the fluxes recovered for the sources for Gaussian versus point-like extractions, which was always $<15 \%$. Our VLA flux measurements of $\mathrm{CO}[1-0]$ are reported in Table 2 .

These measurements are consistent within the errors with those reported in Aravena et al. (2014), but they are not exactly the same and have somewhat smaller uncertainties. This is because by using previously acquired positional, velocity, and size information from the higher- $J$ emission, we were able to reduce some of the uncertainties while also obtaining measurements that are directly comparable with those at higher- $J$, and also because we incorporated the older VLA data. The total VLA integration time on the three BzK galaxies amounts to 259 hours, after accounting for primary-beam reduction (a large part of the data was obtained for the simultaneous observation of GN20 in the $\mathrm{CO}[2-1]$ transition; Hodge et al. 2013).

\subsection{Continuum subtraction and derivation of $\langle U\rangle$}

Given the rapidly rising dust continuum emission with frequency in the Rayleigh-Jeans tail sampled by our data, higher- $J$ $\mathrm{CO}$ emission lines have increasingly lower equivalent width and require a careful continuum subtraction. This is totally negligible for $\mathrm{CO}[1-0]$ and $\mathrm{CO}[2-1]$, marginally important for $\mathrm{CO}[3-2]$, and basically only relevant for $\mathrm{CO}[5-4]$. We obtained a first estimate of the continuum level in the data by averaging all velocity ranges outside the detected $\mathrm{CO}$ emission lines (Table 2; this is slightly different but consistent with what was reported in Table 1 of Magdis et al. 2012). Only upper limits were obtained at $2 \mathrm{~mm}$ below the $\mathrm{CO}$ [3-2] transitions, while some results with a $S / N \sim 2-4$ were found at $1.3 \mathrm{~mm}$. We then refined these low-S/N measurements by exploiting the full IR SED in these galaxies. We used the latest determination of the Herschel fluxes updating measurements shown in Magdis et al. (2012), based on a deblending technique that will be described in forthcoming papers (Daddi et al., in prep.; Liu et al., in prep.), and fitted the SEDs using the models of Draine \& Li (2007), following the approach in Magdis et al. (2011, 2012). Results are shown in Fig. 5. We computed the best-fitting values for the $1.3 \mathrm{~mm}$ and $2.2 \mathrm{~mm}$ continuum flux densities and their uncertainties from the fit (Table 3 ). When we compared this to the measurements, we found a general consistency within the errors. We combined this information (fit and measurement) at $1.3 \mathrm{~mm}$ weighting using their errors to define the best continuum value for subtraction from the $\mathrm{CO}[5-4]$ flux. The associated uncertainties are much smaller than those we obtained from the direct estimate of the continuum to either side of the $\mathrm{CO}$ [5-4] line in the IRAM data alone. At $2.2 \mathrm{~mm}$ we subtracted the flux predicted by the fit as the only available continuum estimate. This correction is small $(\$ 5 \%)$ in all cases. Even if the formal measurements at $2.2 \mathrm{~mm}$ tend to be in excess of the best-fit SED (Fig. 5), we verified that the SED fits are consistent within the errors also when stacking the four sources. 
Table 3. Far-IR SED fitting results and other physical properties.

\begin{tabular}{lcccccccc}
\hline \hline Source & $\langle U\rangle$ & $\log L_{\mathrm{IR}} / L_{\odot}$ & $\log M_{\text {dust }} / M_{\odot}$ & $f_{1.3 \mathrm{~mm}}[\mathrm{mJy}]^{a}$ & $f_{2.2 \mathrm{~mm}}[\mathrm{mJy}]^{a}$ & $\log M_{\text {stars }} / M_{\odot}$ & ${\mathrm{CO} F W H M\left[{ }^{\prime \prime}\right]^{b}}_{c} C^{c}$ \\
\hline 4171 & $16.4 \pm 4.2$ & $12.00 \pm 0.03$ & $8.70 \pm 0.10$ & $0.35 \pm 0.05$ & $0.06 \pm 0.01$ & 10.60 & 1.34 & 0.12 \\
16000 & $4.9 \pm 1.3$ & $11.85 \pm 0.03$ & $9.08 \pm 0.10$ & $0.52 \pm 0.08$ & $0.10 \pm 0.02$ & 10.63 & 1.28 & 0.07 \\
17999 & $13.2 \pm 2.9$ & $12.06 \pm 0.03$ & $8.85 \pm 0.09$ & $0.48 \pm 0.06$ & $0.09 \pm 0.02$ & 10.59 & 0.75 & 0.11 \\
21000 & $15.7 \pm 3.3$ & $12.32 \pm 0.03$ & $9.03 \pm 0.09$ & $0.66 \pm 0.19$ & $0.12 \pm 0.04$ & 10.89 & 0.72 & 0.18 \\
\hline
\end{tabular}

Notes. ${ }^{(a)}$ Fluxes predicted from the SED fitting, computed at rest frequencies of CO[5-4] and CO[3-2]. ${ }^{(b)}$ Reported from D10. ${ }^{(c)}$ Clumpiness measured in the HST-WFC3 F160W images (Sect. 6.6).

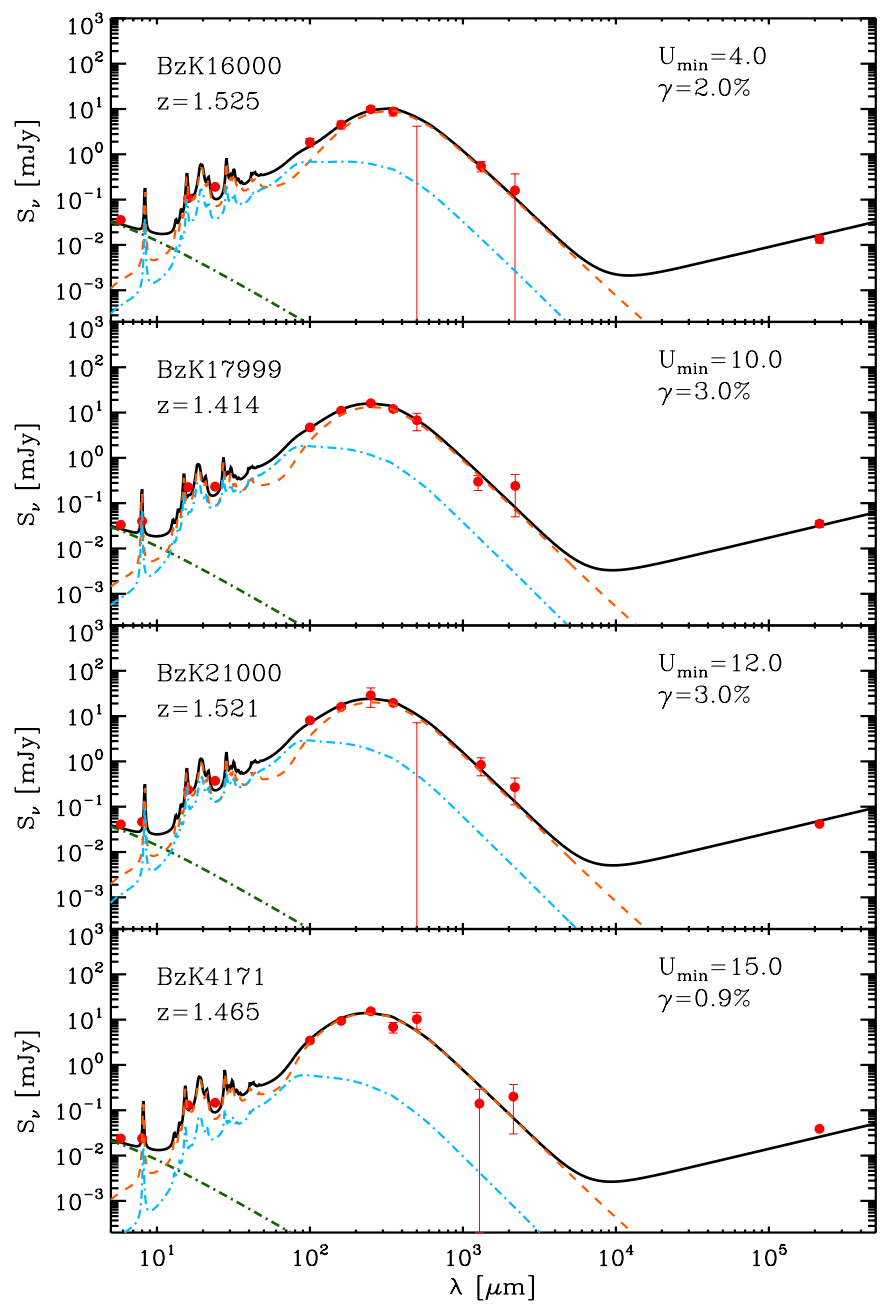

Fig. 5. IR SEDs of our sources, fitted with the models of Draine \& $\mathrm{Li}$ (2007), extended in the radio assuming the local radio-IR correlation. Observed wavelengths are plotted. Orange dashed and cyan dotteddashed lines show separate contributions of starlight and emission from the diffuse ISM component and the PDR component, respectively. The stellar component is shown with a green dotted-dashed line.

These SED fits also allow us to obtain updated estimates of the bolometric $L_{\mathrm{IR}}, M_{\mathrm{dust}}$ and thus mean radiation intensity $\langle U\rangle=1 / P_{0} \times L_{\mathrm{IR}} / M_{\text {dust }}$ for our targets $\left(P_{0}=125 L_{\odot} / M_{\odot}\right.$, so that $\langle U\rangle$ is dimensionless; Draine \& Li 2007). These quantities are reported in Table 3 and are used in the remainder of the paper. They agree within the uncertainties with values published in Magdis et al. 2012 (their Table 2). We recall that $\langle U\rangle \propto T_{\text {dust }}^{4+\beta}$ and, consistently with the findings in Magdis et al. (2012),
Table 4. Average CO SLED of the (U)LIRGs from P12 that we used here.

\begin{tabular}{cccc}
\hline \hline$J$ & $I_{\mathrm{CO}}{ }^{a}$ & $\Delta I_{\mathrm{CO}}{ }^{b}$ & $N_{\text {bands }}{ }^{c}$ \\
\hline 1 & 1.00 & 0.00 & 14 \\
2 & 3.59 & 0.22 & 13 \\
3 & 7.11 & 0.53 & 14 \\
4 & 12.4 & 1.40 & 6 \\
5 & 12.2 & 1.90 & 0 \\
6 & 10.6 & 2.40 & 14 \\
\hline
\end{tabular}

Notes. ${ }^{(a)}$ Fluxes of individual galaxies have been normalised to $\mathrm{CO}[1-0] .^{(b)}$ The error is the rms dispersion of individual measurements divided by the square root of the number of galaxies in the sample. (c) Total number of measurements available for the transition from P12. Lacking a direct measurement, we have used an estimate based on LVG modeling of the other available transitions.

these $\langle U\rangle$ values correspond to dust temperatures in the range of 30-35 K for modified blackbody fits with $\beta \sim 1.5$. The typical uncertainties on $\langle U\rangle$, given the quality of our multi-wavelength dataset, are of about 0.1 dex $(20-30 \%)$. We also show in Fig. 6 target images of the HST Wide Field Camera 3 (WFC3). The F160W imaging, together with the UV rest-frame imaging (see Fig. 5 in D10), reveal reasonably regular morphologies, especially in the rest frame optical, but they are characterized by massive clumps, which is fairly consistent with the typical clumpy disk galaxies at high redshifts (Genzel et al. 2006; Bournaud et al. 2008; Forster-Schreiber et al. 2009).

\subsection{Comparison samples of local spirals and (U)LIRGs}

We used a representative sample of (U)LIRGs taken from from $\mathrm{P} 12$, for which the contribution from star formation is $L_{\mathrm{IR}}>$ $2 \times 10^{11} L_{\odot}$. These authors did not observe CO[5-4] directly, but their sample includes sources with a measurement of $\mathrm{CO}$ [6-5], sometimes including $\mathrm{CO}$ [4-3], and virtually always $\mathrm{CO}$ [3-2], $\mathrm{CO}[2-1]$, and $\mathrm{CO}[1-0]$, see Table 4. For these galaxies we used single-component LVG models (see Sect. 5 for more details on the models) to interpolate between $\mathrm{CO}[6-5]$ and lower- $J$ transitions, but we excluded $\mathrm{CO}[1-0]$ and in some cases $\mathrm{CO}[2-1]$, depending on the fit (consistent results were found with a twocomponent LVG fitting when including the lowest- $J$ transitions). We visually inspected all fits and retained 14 galaxies where the LVG fitting solutions appear to unambiguously return estimates for the $\mathrm{CO}[5-4]$ fluxes. We constructed an average CO SLED of local (U)LIRGs by averaging all available measurements of 

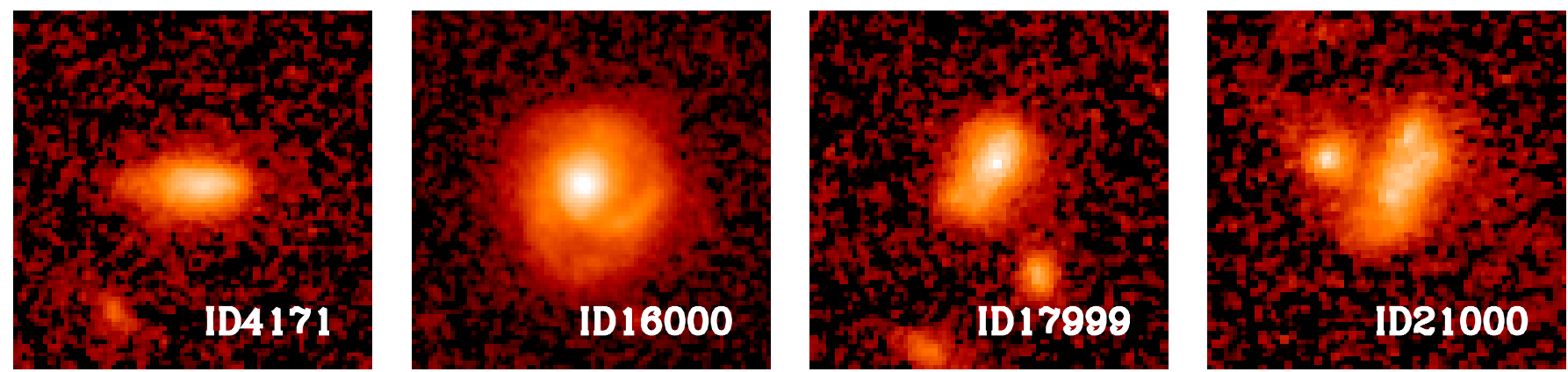

Fig. 6. HST WFC 3 cutouts in the F160W band from public CANDELS imaging (Koekemoer et al. 2011; Grogin et al. 2011). The cutouts are 5" on a side and are shown with logarithmic stretching to improve the visibility of details. The blob to the northeast of BzK-21000 is an unrelated object (north is up and east to the right). For comparison, Fig. 5 in D10 shows three-color HST+ACS imaging of the galaxies.

the 14 galaxies $^{3}$ from P12 and using the modeled value when we lacked measurements. The average (U)LIRGs CO SLED derived in this way is reported in Table 4.

For spiral galaxies we use archival Herschel observations of the $\mathrm{CO}$ [5-4] emission in five Kingfish galaxies ${ }^{4}$ using the SPIRE FTS spectrometer. Observations were performed in mapping mode, and we used data from the central bolometer (36") that cover the nucleus and inner disk of the galaxies. Details of the measurements will be presented in Liu et al. (in prep.). We also used measurements from HERACLES (Leroy et al. 2009) of $\mathrm{CO}[2-1]$. We determined the aperture correction of the Herschel $\mathrm{CO}$ [5-4] measurements using the IRAS imaging. In addition, we used data for the local spiral IC 342 from Rigopoulou et al. (2013) and the MW.

\section{Results}

\subsection{CO SLEDs of normal $z=1.5$ galaxies}

The multitransition CO SLEDs of our four targets are shown in Fig. 7, including CO[1-0] (except for BzK-17999), CO[2-1], $\mathrm{CO}[3-2]$, and $\mathrm{CO}$ [5-4]. We also averaged the fluxes for the four CO transitions for the three BzK galaxies with full SLED observations. Results are shown in Fig. 8, and the fluxes are listd in Table 2. When we compare this with the trend expected for thermally excited $\mathrm{CO}$ emission (red curves, normalized to the lowest- $J$ transition available, with fluxes scaling $\propto J^{2}$ ), we see that generally $\mathrm{CO}[2-1]$ is only weakly subthermally excited. For two galaxies (BzK-21000 and BzK-4171) the CO[2-1] to $\mathrm{CO}[1-0]$ brightness temperature ratio $R_{21}$ (Table 2) is consistent with unity, while BzK-16000 is already significantly subthermally excited at $\mathrm{CO}[2-1]$. As a result, the average $R 21$ for our sample is $0.76 \pm 0.09$, which is moderately subthermally excited. We note that this is formally consistent with the value of 0.86 that we derived in Dannerbauer et al. (2009) and used to correct $\mathrm{CO}[2-1]$ fluxes to $\mathrm{CO}[1-0]$ in D10 and subsequent papers from our group (Daddi et al. 2010b; Magdis et al. 2011, 2012; Sargent et al. 2014, etc.).

Conversely, $\mathrm{CO}$ [3-2] and $\mathrm{CO}[5-4]$ are both significantly subthermally excited in all sources. For the $J=3$ transition we

\footnotetext{
$3 \quad$ IRAS00057+4021, IRAS02483+4302, IRAS04232+1436, IRAS05083+7936-VIIZw031, IRAS05189-2524, IRAS08572+3915, IRAS09320+6134-UGC5101, IRAS10565+2448, IRAS12540+5708Mrk231, IRAS13428+5608-Mrk273, IRAS15327+2340-Arp220, IRAS16504+0228-NGC6240, IRAS17208-0014, and IRAS23365+3604.

4 NGC 3351, NGC 3521, NGC 4736, NGC 6946 and NGC 7331.
}
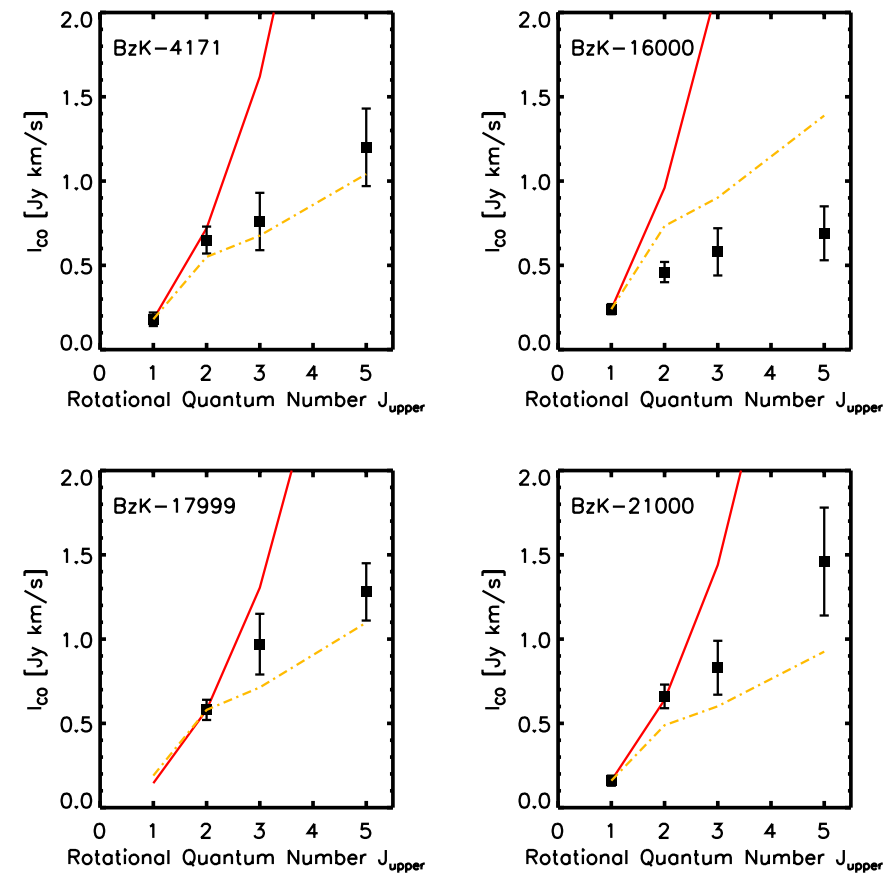

Fig. 7. CO SLEDs for the four BzK galaxies in our sample, including IRAM PdBI data over $\mathrm{CO}$ [2-1], $\mathrm{CO}$ [3-2], and $\mathrm{CO}$ [5-4], and VLA measurements of $\mathrm{CO}[1-0]$ for three galaxies, based on Aravena et al. (2014). The dotted-dashed line shows the average SED for comparison, emphasizing excitation variations within our sample. The solid line corresponds to a constant brightness temperature.

measure an average $R_{31}=0.42 \pm 0.07$, with ranges from 0.27 to 0.57 for individual sources. It is clear that estimating the $\mathrm{CO}[1-0]$ flux and thus total CO luminosities of $z=1.5$ galaxies from $\mathrm{CO}[3-2]$ is less accurate than using $\mathrm{CO}[2-1]$. The typical assumption of $R_{31} \sim 0.5$ that is adopted in the literature (Tacconi et al. 2010, 2013; Genzel et al. 2010, etc.) is consistent with our data on average, however. Globally, the four sources behave quite similarly to BzK-21000, which was first discussed in Dannerbauer et al. (2009; see also Aravena et al. 2010, 2014), and in particular, their SLEDS from $\mathrm{CO}$ [2-1] to $\mathrm{CO}$ [3-2] closely resemble the MW (Fixsen et al. 1999). Presumably, the same holds at $\mathrm{CO}[1-0]$, although the MW data reported by Fixen have a very large error there (see Fig. 7).

Clearly, the average CO SLED deviates strongly from that of the MW and other local spirals at the $\mathrm{CO}[5-4]$ transition: the $\mathrm{CO}$ [5-4] emission of the BzK galaxies is about four times higher on average than what would be expected from normalizing the 


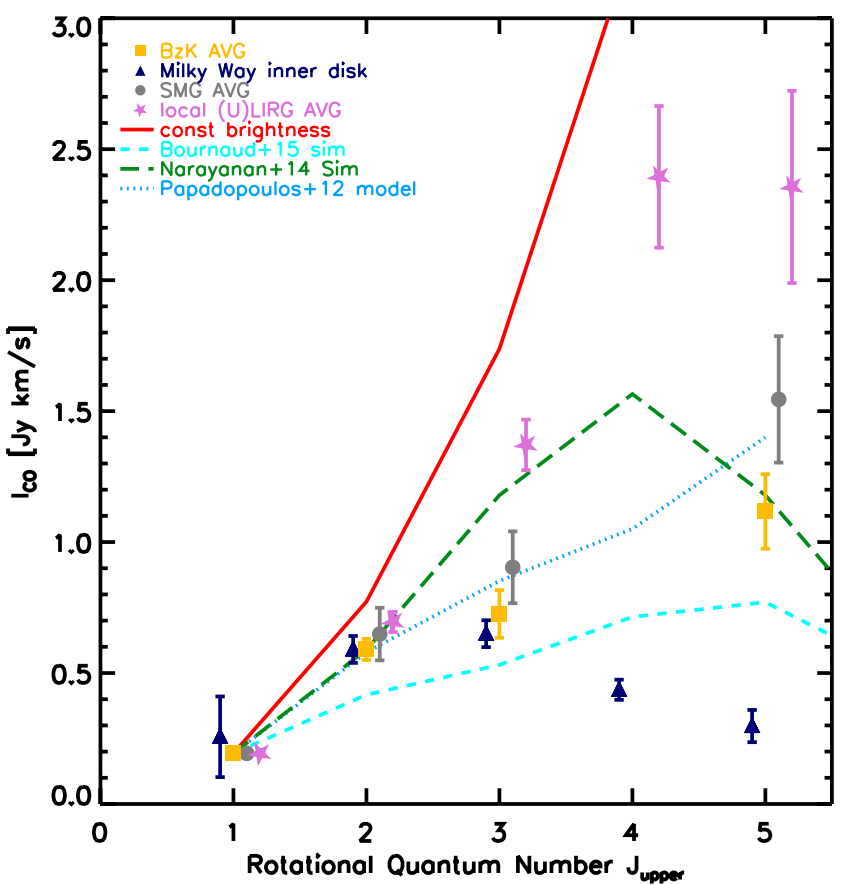

Fig. 8. Comparison of the average SLED of BzK galaxies to the MW SLED (Fixsen et al. 1999), the average of SMGs (Bothwell et al. 2013; the SLED reported by Spilker et al. (2014) is similar, but higher at $J>5$ ) and the average (U)LIRGs SLED derived in this paper using measurements from P12. All SLEDs are normalized to the $\mathrm{CO}[1-0]$ of the average BzK galaxy SLED, except the MW, which is normalized using $\mathrm{CO}[2-1]$. The results from the toy model of P12 (see text) and the numerical simulations of B15 and Narayanan \& Krumholz (2014) are also shown.

MW SLED to the $J \leq 3$ transitions. We see CO[5-4] flux in excess of MW expectations in all cases, although for BzK-16000 the excess is the lowest, only of a factor of two. The average $R_{51}$ is $0.23 \pm 0.04$, with ranges for individual sources extending from 0.12 to 0.36 , or about a factor of 3 . This suggests that extrapolating $\mathrm{CO}[1-0]$ fluxes from $\mathrm{CO}[5-4]$ for this population would likely lead to uncertainties of about factors of two.

Globally, the BzK galaxies have CO SLEDs that are less excited than those of local (U)LIRGs. On average, P12 (U)LIRGs have a ratio of $\mathrm{CO}[3-2] / \mathrm{CO}[2-1]>0.8$ for their sample, compared to 0.52 in our case. Similarly, we find that starbursting local (U)LIRGs have flux ratios of $\mathrm{CO}[5-4] / \mathrm{CO}[2-1]$ higher by a factor of 1.8 .

Comparing these results to SMGs, for which Ivison et al. (2011) reported $R_{31}=0.55 \pm 0.05$ and Bothwell et al. (2013) $R_{31}=0.52 \pm 0.09$, we see that the normal MS galaxies at $z=1.5$ appear to have somewhat less excited CO SLEDs $(R 31=0.42 \pm 0.07)$. Similarly, Bothwell et al. (2013) reported $R_{51}=0.32 \pm 0.05$, which is also higher than our average value $(0.23 \pm 0.04)$, while the spectrum obtained by Spilker et al. (2014) of gravitationally lensed sources discovered with the South Pole Telescope (SPT) has even higher ratios, implying that the flux ratios of $\mathrm{CO}[5-4] / \mathrm{CO}[1-0]$ for SMGs are higher by $40 \%$ or more on average. This is confirmed by the comparison of the global SED in Fig. 8, which shows a higher excitation for the SMGs at $J \geq 3$. We note from Fig. 8 that, in comparison, the CO SLED of SMGs is intermediate in excitation strength between that of local (U)LIRGs and $z=1.5 \mathrm{BzKs}$. This supports the notion that SMGs are not purely SBs, but probably a fair mix of MS and SB populations (see, e.g., Rodighiero et al. 2011), although the higher excitation of the SPT sample might also be connected to their higher average redshift (see next sections). There are individual SMGs with a CO excitation comparable to that of the BzK galaxies (e.g., Riechers et al. 2011).

\subsection{Comparison to theoretical predictions of CO SLEDs}

In Fig. 8 we compare the average SLED of the BzK galaxies with several predictions for high-redshift disks from theoretical modeling, which all agree fairly well with the data, at least in a qualitative sense. These models correctly predict an enhancement over the MW CO SLED, although complete agreement with the average BzK data is not achieved by any model at this stage.

P12 (blue dotted line) provide predictions for high- $z$ BzK galaxies analogous to our sources based on simple reasoning and assuming a scaling of the $\mathrm{CO}$ excitation depending on the SFE or equivalently the fraction of dense gas (assuming a universal SFE per dense molecular gas mass). In their interpretation, the high- $J \mathrm{CO}$ emission is heated by turbulence and/or cosmic rays in the dense gas (e.g., Papadopoulos et al. 2014). Their expected SLEDs are not far from the observed one, but rise more rapidly than the data at the highest transitions. It seems plausible that full agreement with the observations might be obtained by tuning some of the free parameters for which they could only provide guesses.

More recently, Narayanan \& Krumholz (2014) have developed a general model for predicting CO observations over different transitions by combining numerical simulations and radiative transfer calculations. In their case, the overall excitation is regulated by the star formation surface density $\left(\Sigma_{\mathrm{SFR}}\right.$; a similar behavior is predicted by Popping et al. 2014). Based on the typical $\Sigma_{\mathrm{SFR}} \sim 1 M_{\odot} \mathrm{yr}^{-1} \mathrm{kpc}^{-2}$ for BzKs and normal $z=1-2$ disk galaxies (Daddi et al. 2010b; Genzel et al. 2010), we can use their predictions to compare the results with BzK galaxies (green line in Fig. 8). The Narayanan \& Krumholz (2014) model correctly predicts enhanced $\mathrm{CO}[5-4]$ emission compared to the MW (Fig. 8), although with an overall shape that is somewhat different from that of the BzKs, rising steeply toward $\mathrm{CO}[4-3]$ and resulting in too high ratios of $\mathrm{CO}[3-2] / \mathrm{CO}[2-1]$. It appears that the CO SLED of BzK galaxies has an unexpected shape, fairly flat and MW-like up to $\mathrm{CO}[3-2]$ and rising quite rapidly afterwards, giving peculiarly high ratios of $\mathrm{CO}[5-4]$ (or $\mathrm{CO}$ [4-3]) to $\mathrm{CO}[3-2]$ (the agreement with a MW shape up to $J=3$ is correctly anticipated by P12).

Finally, we compare our results with the predictions of CO SLEDs for high- $z$ disk galaxies by B15, based on very high resolution AMR numerical simulations. In this case, the models also predict a more highly enhanced $\mathrm{CO}[5-4]$ emission than for the MW, although the discrepancy here is that the expected SLED is less excited than observed, with expected $R_{51} \sim 0.14$. In the B15 simulations the high- $J$ excitation component of the CO SLED is mainly attributed to the giant clumps, while the rest of the diffuse SF in the galaxy contribute a low- $J$ excitation component. In this respect, the $\mathrm{CO}$ excitation is lower than observed because the gas fraction is underestimated compared to the real galaxies, because a lower gas fraction will result in fewer and less massive clumps in the galaxies (see Salmi et al. 2012; Bournaud et al. 2012, 2011). The low gas fraction arises because the simulations start with a (reasonable) gas fraction of $\sim 50 \%$ (albeit this is for the total gas, the molecular gas fraction might be even lower), but given the lack of gas accretion in the simulations during the lifetime of the galaxy, the gas fraction decreases 
and is less optimal. Another explanation for the lower $R_{51}$ in the models might be that resolution effects bias the computation of CO[5-4] emission low (B15). In any case, B15 successfully predicted a difference in the CO SLED between local (U)LIRGs and high- $z$ disks, which qualitatively agrees with the observations.

Overall, the model of B15 provides too little flux in all lines above $\mathrm{CO}[1-0]$ (or, equivalently, too strong $\mathrm{CO}[1-0]$ emission), while the model of Narayanan \& Krumholz predicts a SLED shape different from the data. The P12 model provides too much flux at $J \geq 3$ but performs somewhat better that the other models. When we examine individual sources rather than the average SLED, we see that the BzK galaxy with the highest $\mathrm{CO}$ excitation (BzK-21000) is consistent with the expectations of P12. The high- $J$ transitions of the galaxy with the lowest excitation, BzK-16000, are even less excited than the significantly subthermal excitation predicted by the simulations of B15.

\section{LVG modeling}

Large velocity gradient (LVG) modeling is a classical tool for studying CO SLEDs and gaining insights into the physical properties of the gas, including their kinetic temperature and density ( $T_{\text {kin }} ; n$; Goldreich \& Kwan 1974; Scoville \& Solomon 1974). We followed the approach described in Weiss et al. (2007), Dannerbauer et al. (2009) and Carilli et al. (2010), using the collision rates from Flower (2001) with an ortho-to-para ratio of 3 and a $\mathrm{CO}$ abundance per velocity gradient of $[\mathrm{CO}] / \Delta v=$ $1 \times 10^{-5} \mathrm{pc}\left(\mathrm{km} \mathrm{s}^{-1}\right)^{-1}$. We computed model grids using an appropriate value of the $\mathrm{CMB}$ temperature for $z=1.5$ and varying the density over $10^{2}-10^{6} \mathrm{~cm}^{-3}$ and $T_{\text {kin }}$ over 5 to $100 \mathrm{~K}$.

Single-component models are inadequate for fitting the whole SLED of the BzK galaxies, as is obvious from the broad shape with a fairly flat flux level from $\mathrm{CO}[2-1]$ to $\mathrm{CO}[5-4]$. This is true for the composite SLED and also for individual sources, except for BzK-17999, which can be formally fit by a single component, but probably only as a result of the lack of $\mathrm{CO}[1-0]$ constraints. On the other hand, two-component models are highly degenerate, and a wide variety of acceptable solutions can be found. This is at least in part due to the intrinsic degeneracies of this type of modeling, where increasing density or temperature affects the SLEDs in the same way at least to first order, but also because measured fluxes continue to rise all the way to $\mathrm{CO}[5-4]$, and we have no evidence of where beyond this transition the SLED begins to decrease. In Fig. 9 we show a map of $\chi^{2}$ values as a function of the density and temperature, separately for a low-excitation and a high-excitation two-component fit. It appears that a MW-like low-excitation component with $\log n / \mathrm{cm}^{-3} \sim 2-3$ and moderate temperature $T_{\text {kin }} \sim 20-30 \mathrm{~K}$, which is similar to the dust temperature in these galaxies (Magdis et al. 2012; Sect. 3.3), together with a denser and/or warmer component can fit the data well. Other and different viable solutions exist as well, obviously, but good $\chi^{2}$ regions appear to constrain the density of the two components more stringently, with the low-excitation component preferring $\log n \gtrsim 3$ and the high-excitation component having $\log n \gtrsim 3.5$, at least when the solutions are limited by $T_{\text {kin }}<100 \mathrm{~K}$, as we have assumed. Allowing for temperatures substantially higher than $100 \mathrm{~K}$ in the high-excitation component would reduce the lower limit on its density, as can be guessed from the trends in Fig. 9 (top right panel).

Overall, these results are consistent with the simulations of B15, who suggested that the high-excitation component (mainly related to the giant clumps) is denser but also warmer than the diffuse gas, and its particularly high temperature $(100 \mathrm{~K}$ for $n=3300 \mathrm{~cm}^{-3}$ in a $\mathrm{CO}[5-4]$ luminosity weighted average of the molecular gas in B15 - an estimate which might still retain some contamination from diffuse gas) is key in driving its strongly peaked shape toward $\mathrm{CO}$ [5-4]. Their predicted values are reasonably consistent within the range of acceptable values from our modeling, although our constraints on $T_{\text {kin }}$ are not very stringent and the B15 predicted density is on the low-side of the acceptable values for the high excitation component.

In Fig. 10 we show particular LVG solutions obtained by fitting the data with reasonable $\chi^{2}$ values, which were chosen to display a peak of the low-excitation component at $J \sim 2$ and at $J \sim 5$ (left panel) or $J \sim 7$ (right panel) for the high-excitation component. Representative density and kinetic temperature values for the adopted solutions are shown in the figure caption. This kind of behavior, as in the left panel of Fig. 10, with an overall peak of the CO SLED not far from $\mathrm{CO}$ [5-4], might be expected from the simulations of B15 and Narayanan \& Krumholz (2014). The calculations of P12 would suggest a peak at even higher- $J$, similar to the warmest among local LIRGs (e.g., Lu et al. 2014).

LVG modelling can be used to estimate the molecular gas mass of the CO-emitting galaxies, basically exploiting the constraints on the gas densities. This requires critical assumptions on key physical parameters, however, which enter the calculation of the CO SLEDs at various luminosities for a given gas mass, namely: the disk scale height (or turbulence velocity of the medium) and the $\mathrm{CO}$ abundance per velocity gradient. Hence final estimates are highly uncertain, as direct constraints on this parameters are not very accurate. We verified that by using plausible estimates for these parameters, we can match the gas mass estimates of about $5-8 \times 10^{10} M_{\odot}$ that are typically inferred for these galaxies using dynamical methods (D10), or converting from dust mass estimates (Magdis et al. 2011, 2012), or assuming a local metallicity scaling for the $\alpha_{\mathrm{CO}}$ conversion factor (Sargent et al. 2014). Moreover, typical LVG solutions suggest that a non-negligible fraction of the total gas mass resides in the more excited component, in some cases, this reaches half of the total gas. This is consistent with the predictions of B15.

\section{Interpretation and discussion}

\subsection{What is the main driver of CO excitation?}

We have shown that $\mathrm{CO}[5-4]$ emission inside normal MS galaxies at $z=1.5$ is substantially excited. This result is markedly different from what is seen in local spirals and the MW. There are a number of reasons why this could be expected, as anticipated in the introduction to this paper: the rising dust temperature in MS galaxies as reflected by the $\langle U\rangle$ parameter (e.g., Magdis et al. 2012), the higher fraction of dense gas and SFE (e.g., Daddi et al. 2010b), the harder ionizing radiation field (Steidel et al. 2014), the probably higher average gas density. Similarly, models appear to suggest different alternatives as the driver of higher excitation gas in high- $z$ BzK galaxies: the SFE (P12), the $\Sigma_{\text {SFR }}$ (Narayanan \& Krumholz 2014) or the warm and dense gas in the clumps (B15). It is therefore relevant to try to gain insights into the different possibilities using our observations. We have collected measurements of the $\mathrm{CO}[5-4] / \mathrm{CO}[2-1]$ flux ratios of various relevant galaxy populations from the literature and from publicly available observations. As anticipated in Sect. 4, for local starbursts we find an average flux ratio of $\mathrm{CO}[5-4] / \mathrm{CO}[2-1]$ of 3.5 with an error on average of 0.09 dex inferred from the dispersion of the (U)LIRG measurements, while for local spirals we find an average ratio of 0.7 with an error of 0.05 dex. 

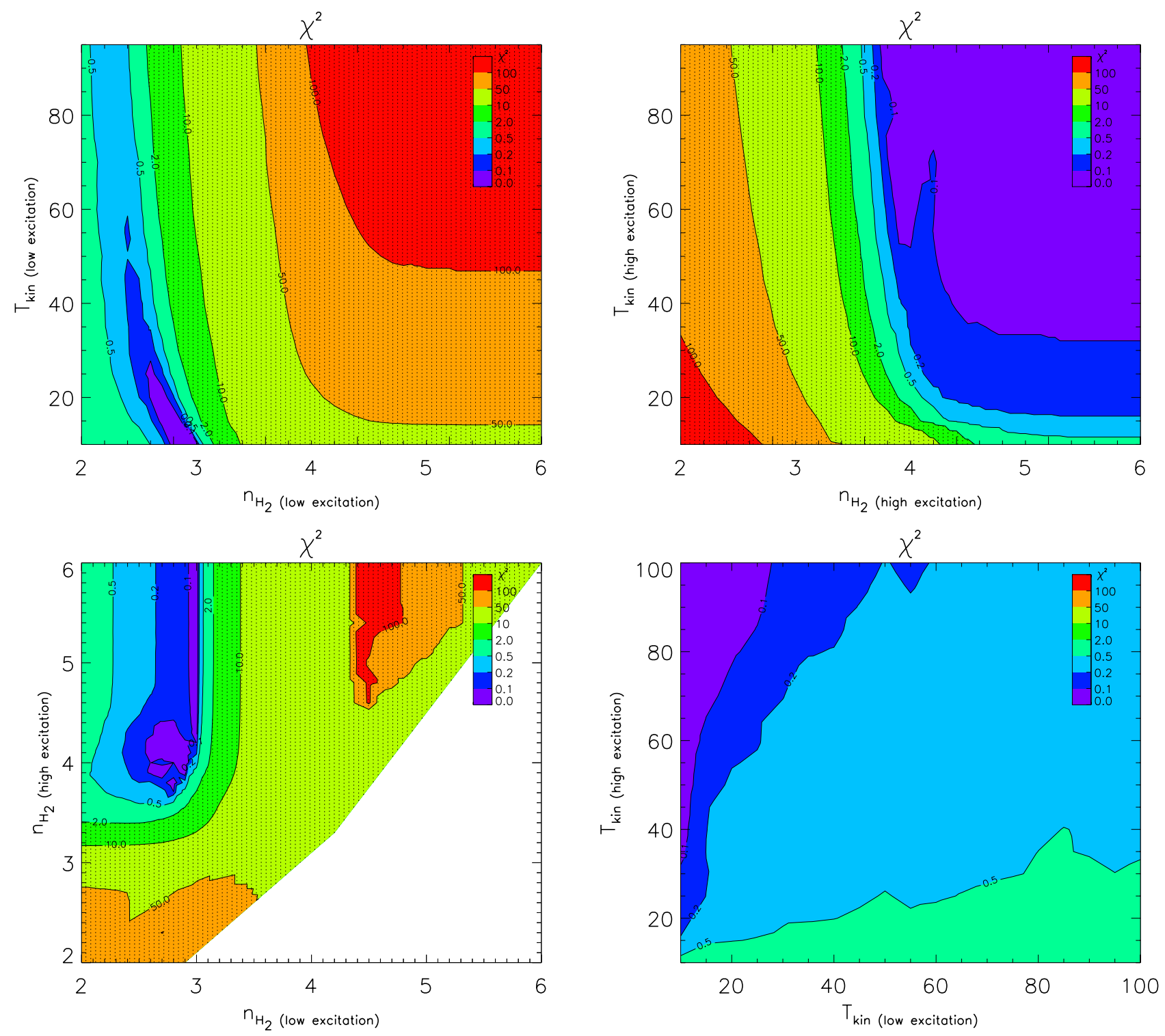

Fig. 9. Total $\chi^{2}$ maps versus temperature and density for the fit of the BzK average CO SLED with two components (a low-excitation and a high-excitation component, the latter defined to peak at higher $J$ ). The color scale reflects the $\chi^{2}$ level: blue and light green regions correspond to acceptable fits. For a choice of variables in each panel, the minimum $\chi^{2}$ is computed by marginalizing over the other two free parameters.

In Fig. 11 we compare the average $\mathrm{CO}[5-4] / \mathrm{CO}[2-1]$ of these three key populations to their average $\langle U\rangle$, SFE and $\Sigma_{\text {SFR }}$. The $\langle U\rangle$ values for the four BzK galaxies were updated in this work and are shown in Table 3 with their error bars. For the spiral galaxies we fitted their IR SEDs using the models of Draine \& Li (2007) to the photometry provided by the Kingfish project (Dale et al. 2012), in analogy to the procedures used for the BzK galaxies (see Magdis et al. 2011, 2012). We use here and in the following only objects for which $\langle U\rangle$ could be measured, which limits the sample to six objects in total. For the (U)LIRGs we used the average $\langle U\rangle$ value of 35 from Magdis et al. (2012) and da Cunha et al. (2010) because individual measurements are available for only three galaxies $(\langle U\rangle=32.5,24.6$ and 52.8 for IRAS10565, IRAS17208 and IRAS12112, returning an average that is also very close to the adopted value for this smaller subsample). To estimate typical values for the SFE, we adopted the values reported in Sargent et al. (2014). The adopted values, reported in the figure, depend on our current understanding of the determination of total gas masses. This is based on $\mathrm{CO}$ measurements with a metallicity dependent $\alpha_{\mathrm{CO}}$ for local spirals and dynamically derived $\alpha_{\mathrm{CO}}$ for local ULIRGS, while for BzK galaxies they are derived by concordant estimates based on CO luminosities and dust mass measurements. Values are constrained to within typical ranges less than a factor of 2 , but we adopted a larger error for ULIRGs to account for the substantial spread in the population. The $z=1.5 \mathrm{BzK}$ galaxies have a SFE only twice higher than local spirals, which is consistent with the small tilt of the KS relation for MS galaxies, while (U)LIRGs have about ten times the SFE of BzK galaxies. We note that the average $L_{\mathrm{IR}}$ in the (U)LIRG sample used here is consistent with the average derived in Sargent et al. (2014). For $\Sigma_{\mathrm{SFR}}$ we used sizes measured by Leroy et al. (2008) for spirals, for (U)LIRGs we used measurements from Kennicutt (1998), Scoville (1994), and Downes \& Solomon (1998), and for BzK galaxies we used the optical sizes (D10, Table 3).

It is quite instructive to compare the three panels of Fig. 11. Good correlations of the ratio $\mathrm{CO}[5-4] / \mathrm{CO}[2-1]$ are found for the $\langle U\rangle$ parameter (left panel) and the $\Sigma_{\mathrm{SFR}}$. However, local 

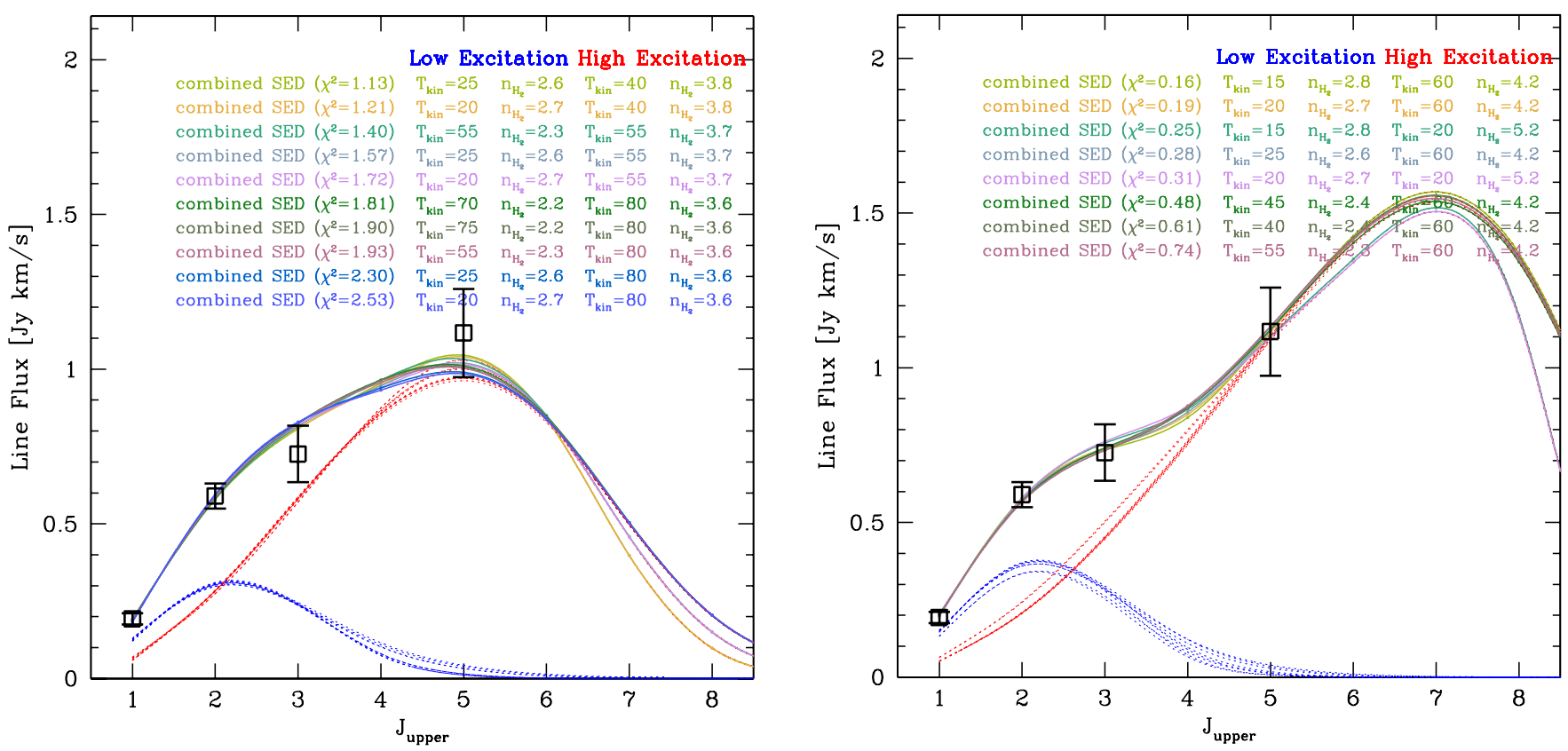

Fig. 10. Examples of LVG fits to the average CO SLEDs of BzK galaxies. These particular solutions (among the large suite of acceptable ones) were chosen to have a cold component peaking at $J \sim 2$ and a warmer and denser component peaking at $J \sim 5$ (left panel) and at $J \sim 7$ (right panel). The captions show details of the physical parameters of each solution. Solutions peaking at higher $J$ obviously exist and can also fit the data well.

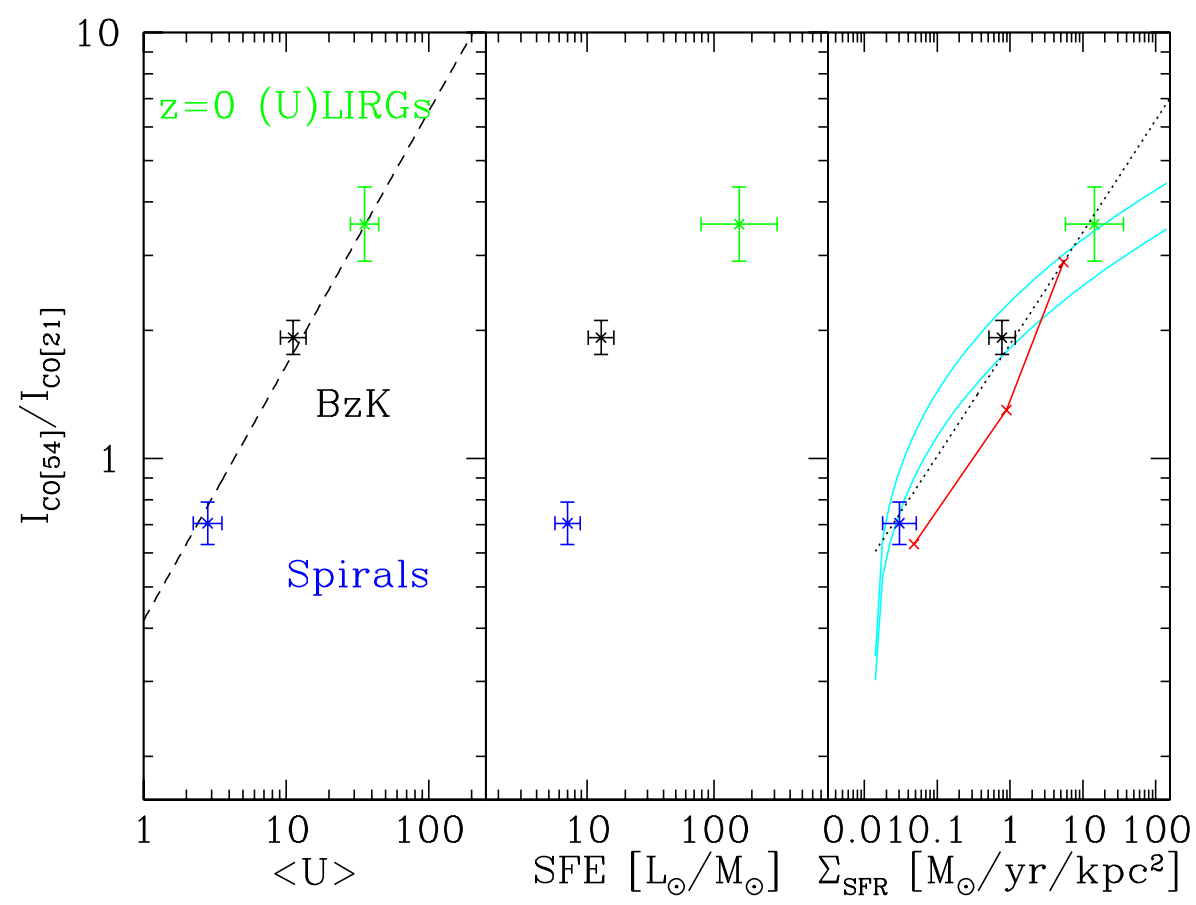

Fig. 11. Comparison of the average $\mathrm{CO}[5-4] / \mathrm{CO}[2-1]$ ratio to the average radiation field intensity $(\langle U\rangle$, left panel), the star formation efficiency (SFE, center panel), and the star formation surface density ( $\Sigma_{\mathrm{SFR}}$; right panel), for local spirals (blue), local (U)LIRGs (green), and $z=1.5 \mathrm{normal}$ MS galaxies (black). The best-fitting trend (dashed line) in the left panel is given in Eq. (1), while the relation in the right panel (dotted line) is given in Eq. (3). The cyan trends (right panel) show the predictions of Narayanan \& Krumholz (2014) based on their Eq. (19) and Tables 2 and 3, while in red we show the B15 simulation results.

spirals and $z=1.5 \mathrm{MS}$ galaxies have similar SFEs (center panel), so that it is difficult to explain the stronger $\mathrm{CO}[5-4]$ emission in BzK galaxies only with their higher SFE, also compared to the location of (U)LIRGs, for example. The correlation between $\mathrm{CO}$ excitation and $\Sigma_{\mathrm{SFR}}$ agrees fairly well with predictions of the model by Narayanan \& Krumholz (2014) and also displays a trend similar to the predictions of B15.

\subsection{A sublinear correlation of $\mathrm{CO}$ excitation with $\langle U\rangle$}

We explore the correlation between the ratio of $\mathrm{CO}[5-4] /$ $\mathrm{CO}[2-1]$ and $\langle U\rangle$ in more detail in Fig. 12, where we now show individual measurements for spiral galaxies and BzK-galaxies. We also add here a sample of SMGs for which $\langle U\rangle$ has been measured by Magdis et al. (2012) and that are classified as 


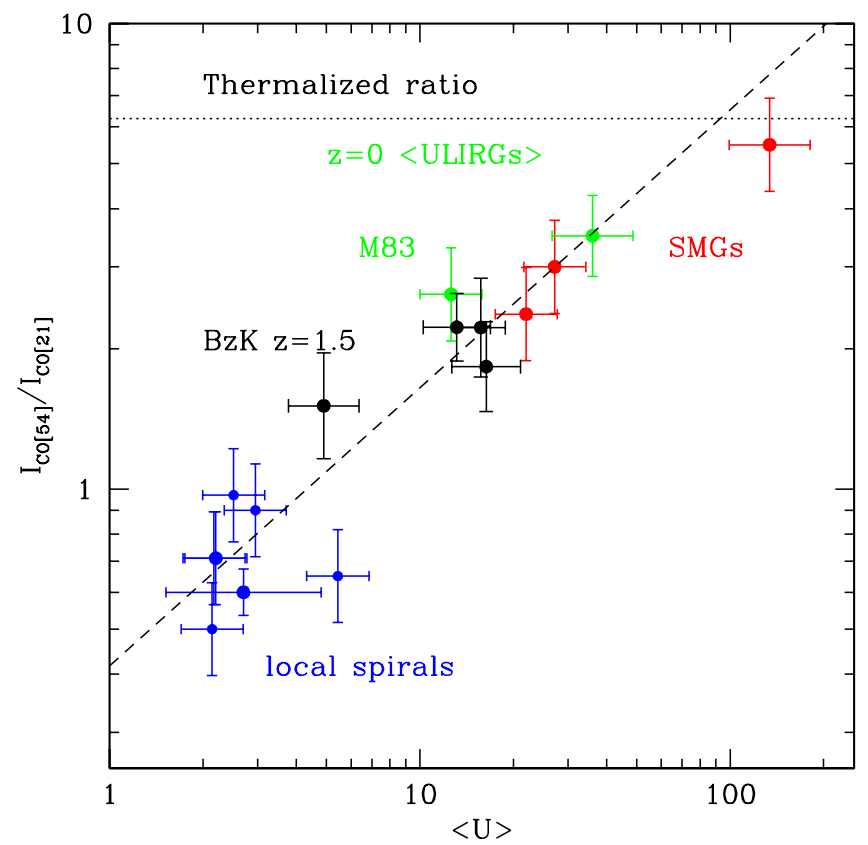

Fig. 12. Ratio of $\mathrm{CO}[5-4]$ to $\mathrm{CO}[2-1]$ flux versus the average radiation intensity $\langle U\rangle$ for the four BzK galaxies. They are compared to the MW and a sample of local spirals from Kingfish (Dale et al. 2012) observed with Herschel FTS (Liu et al., in prep.), to the average of (U)LIRGs from P12 and the M83 starburst (Wu et al. 2015), and to starburst-like SMGs. The horizontal line shows the limiting flux ratio of $\mathrm{CO}[5-4]$ to $\mathrm{CO}[2-1]$ expected for a constant brightness temperature. The dashed line shows the best-fitting trend to the data (Eq. (1)).

starburst galaxies. (We recall that general SMG samples are instead probably mixed ensembles of MS and off-MS SB galaxies, e.g., Rodighiero et al. 2011.) These include GN20, for which the CO measurements are taken from Carilli et al. (2010), SMM-J2135 (cosmic eye) from Danielson et al. (2011), and HSLW-01, for which we take CO measurements from Scott et al. (2011). The latter two are lensed sources, and we used de-lensed luminosities for this work. We also added the low-luminosity starburst M83, taking the measurements from Wu et al. (2015). We still used an average $\langle U\rangle$ value for the (U)LIRGs because there are too few individual measurements. The data are best fit by the relation

$\log I_{\mathrm{CO}[5-4]} / I_{\mathrm{CO}[2-1]}=0.60 \times \log \langle U\rangle-0.38$,

with a formal uncertainty in the slope of 0.07 and a dispersion in the residual of only $0.11 \mathrm{dex}$ (a $30 \%$ accuracy in predicting the ratio of $\mathrm{CO}[5-4] / \mathrm{CO}[2-1]$ based on $\langle U\rangle)$. The relation is thus substantially sublinear. We estimate that for the ratio of $\mathrm{CO}[5-4] / \mathrm{CO}[1-0]$ the correlation with $\langle U\rangle$ would steepen to a slope of $\sim 0.7$ (because of the systematically rising $R_{21}$ from spirals to BzK galaxies to (U)LIRGs), but would remain sublinear. A similar correlation between the ratio of high- $J$ to low- $J$ CO and $\langle U\rangle$ was found by $\mathrm{Wu}$ et al. (2015) for resolved SF regions in NGC253. It is quite interesting to recall that following Magdis et al. (2012), we can expect that $\langle U\rangle \propto S F E / Z$ for our sources. Therefore $\langle U\rangle$ can be directly related to SFE in this analysis. However, as discussed earlier in this section, the SFE of local spirals and BzK galaxies is quite similar and cannot justify the different levels of CO excitation. Hence we conclude that metallicity $(Z)$ differences probably account for an important part of the difference in excitation between local spirals and $z=1.5$ BzK galaxies. Although we do not have direct metallicity estimates at hand for our targets, we recall that at fixed mass the metallicity of typical SF galaxies decreases by over a factor of two from $z=0$ to 2 (see, e.g., Erb et al. 2006; Steidel et al. 2014; Maier et al. 2014; Zahid et al. 2014; Valentino et al. 2015). However, metallicity probably only plays an indirect role: as a result of lower gas-do-dust ratios and reduced shielding, it affects the intensity and hardness of the radiation field (and in particular $\langle U\rangle$ ). The stronger radiation field (manifesting as higher dust temperatures) then probably directly affects the $\mathrm{CO}$ excitation. Basically, high $\langle U\rangle$ values and thus higher $\mathrm{CO}$ excitation than in local spirals are derived either because of the large SFE as in the (U)LIRGs, or because of the combined role of SFE and $\mathrm{Z}$ (as in high- $z$ MS galaxies).

\subsection{Dependences on the SFR surface density}

We also provide some further information on the relation between the $\mathrm{CO}[5-4] / \mathrm{CO}[2-1]$ ratio and $\Sigma_{\mathrm{SFR}}$. The trend reported in Fig. 11 (right panel, dotted line) scales as

$\log I_{\mathrm{CO}[5-4]} / I_{\mathrm{CO}[2-1]}=0.26 \times \log \left(\Sigma_{\mathrm{SFR}}\right)+0.27$,

with $\Sigma_{\mathrm{SFR}}$ in the usual units of $M_{\odot} \mathrm{yr}^{-1} \mathrm{kpc}^{-2}$. The correlation between $\mathrm{CO}$ excitation and $\Sigma_{\mathrm{SFR}}$ agrees qualitatively with the predictions of the model of Narayanan \& Krumholz (2014), but their calculations for $\Sigma_{\mathrm{SFR}} \sim 1$ (as in BzK galaxies) results in a CO SLED that is somewhat different in shape than that of the average BzK galaxy. In Fig. 11 (right panel, cyan line) we explicitly report the prediction for the dependence of the ratio of $\mathrm{CO}[5-4] / \mathrm{CO}[2-1]$ on $\Sigma_{\mathrm{SFR}}$, which appears to compare nicely to the trend reported in Eq. (3).

Therefore, it appears that $\Sigma_{\mathrm{SFR}}$ can be used as a good tracer of $\mathrm{CO}$ excitation, at least in our sample including local spirals and (U)LIRGs and high redshift disks. This is not surprising, given the results in the previous sections coupled with the well established correlation between dust temperature (i.e., $\langle U\rangle$ ) and $\Sigma_{\text {SFR }}$ (Chanial et al. 2007; Hwang et al. 2010; Elbaz et al. 2011). Explicitly, within our sample the correlation of $\Sigma_{\mathrm{SFR}}$ with $\langle U\rangle$ is observed as:

$\log \langle U\rangle=0.41 \times \log \left(\Sigma_{\mathrm{SFR}}\right)+1.08$

which confirms the idea that "compactness", equivalent to $\Sigma_{\mathrm{SFR}}$, can be also seen as a key parameter driving the physical properties of galaxies (Elbaz et al. 2011; Rujopakarn et al. 2011).

\subsection{A linear correlation between $L^{\prime} C O[5-4]$ and $L_{\mathrm{IR}}$ ?}

The availability of $\mathrm{CO}[5-4]$ measurements for normal galaxies at $z \sim 1.5$ prompts us to re-assess the correlation between CO luminosities and $L_{\mathrm{IR}}$ using such a transition. We recall that the first observations of low- $J$ CO in MS galaxies at $z \sim 1-2$ (Daddi et al. 2008, 2010; Tacconi et al. 2010) allowed the insight that such objects have SFRs similar to (U)LIRGs but ratios of $L^{\prime} \mathrm{CO}[1-0] / L_{\mathrm{IR}}$ on average three times higher. The substantially higher ratio of $\mathrm{CO}[5-4] / \mathrm{CO}[2-1]$ in (U)LIRGs suggests that at $\mathrm{CO}[5-4]$ one might find similar ratios of $L^{\prime} \mathrm{CO}[1-0] / L_{\mathrm{IR}}$ for (U)LIRGs and BzK galaxies. We show the correlation between $L^{\prime} \mathrm{CO}[5-4]$ and $L_{\mathrm{IR}}$ in Fig. 13 using the galaxy samples discussed in the previous section, but exploiting individual measurements here for (U)LIRGs from P12, as opposed to only considering an average value, as in Fig. 12. We also benefit for this analysis from an enlarged sample of 16 additional SMGs taken from the compilation of Carilli \& Walter (2013), and we used the full sample of (U)LIRGs (selected with $L_{\mathrm{IR}}>10^{11.3} L_{\odot} ; 40$ objects in addition to the P12 sample) with Herschel/SPIRE/FTS archive 


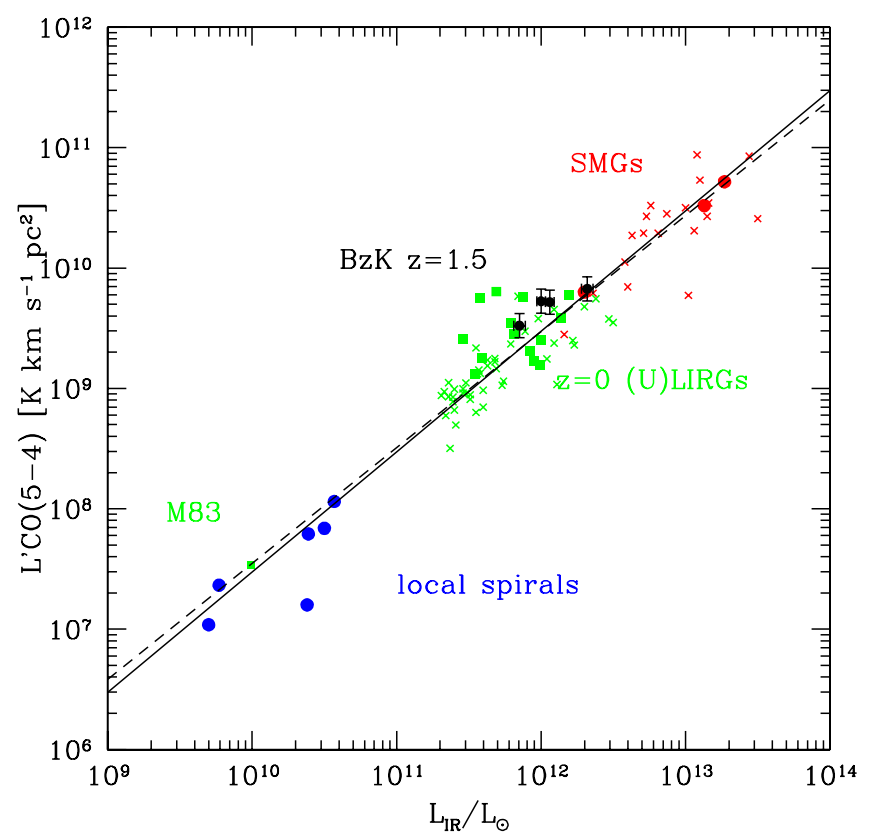

Fig. 13. Correlation of the $\mathrm{CO}[5-4]$ line luminosity with the bolometric IR luminosity $\left(L_{\mathrm{IR}} ; 8-1000 \mu \mathrm{m}\right)$ of our four BzK galaxies at $z=1.5$. When we include the sample of galaxies from Fig. 11 (solid symbols) and additional SMGs and (U)LIRGs (crosses; see text), we observe a virtually linear correlation between the luminosities (solid line, while the dashed line is the nonlinear fit), spanning about four orders of magnitude along both axes. The average $L_{\mathrm{CO}[5-4]}^{\prime} / L_{\mathrm{IR}}$ ratio of the BzK galaxies is consistent with the average of the ratio of local (U)LIRGs or high- $z$ starbursting SMGs.

data that will be presented in Liu et al. (in prep.). Quite strikingly, the formal best-fitting relation has a slope of $0.96 \pm 0.04$ and it is thus indistinguishable from the linear trend

$\log L_{\mathrm{CO}[5-4]}^{\prime}=\log L_{\mathrm{IR}} / L_{\odot}-2.52$

(for $L^{\prime} \mathrm{CO}$ in the usual units of $\mathrm{K} \mathrm{km} \mathrm{s}^{-1} \mathrm{pc}^{2}$ ), with a dispersion in the residual of $0.24 \mathrm{dex}$, which is remarkable for a relation spanning four orders of magnitude along either axis. We note in particular that for BzK galaxies and P12 (U)LIRGs we find fully consistent luminosity ratios of $-2.36 \pm 0.05$ and $-2.34 \pm 0.08$, respectively (we quote the error on the averages, and values are in the units of Eq. (4)). Both populations have somewhat higher ratios than the average, while spirals have $-2.67 \pm 0.13$, which is somewhat lower and affected by the strong outlier NGC 6946. We are limited, however, by small number statistics, and within the current uncertainties, we cannot detect significant differences among the various populations. A similar conclusion about a seemingly linear slope between $L^{\prime} \mathrm{CO}[5-4]$ and $L_{\mathrm{IR}}$ between local (U)LIRGs and high- $z$ SMGs is also presented in Greve et al. (2014), although the novelty here is that we can situate the normal MS galaxies at $z=1.5$ in this relation and that we also include local spirals (see also Liu et al., in prep.).

The basically linear correlation between $L^{\prime} \mathrm{CO}[5-4]$ and $L_{\mathrm{IR}}$ is different from findings for low- $J \mathrm{CO}$, where considering all galaxies together yields a trend more consistent with a slope of 1.7 (e.g., see the classical work of Solomon \& van der Bout 2005) or, when distinguishing SB and MS galaxies, separate relations with slopes of 1.2 and different normalizations (e.g., Sargent et al. 2014). This suggests that CO[5-4] could behave as a tracer of the star-forming gas, meaning that the gas is dense enough to be directly related to the amount of stars formed, in a way that is insensitive of whether the star formation occurs in a quiescent disk or inside a violent merger. If this is the case, then $\mathrm{CO}[5-4]$ could be used as a more efficient and accessible tracer of the dense gas than standard molecules such as HCN or HCO, which also scale basically linearly with $L_{\mathrm{IR}}$, but are intrinsically 10-20 times fainter and harder to observe (Gao \& Solomon 2004a,b; Gao et al. 2007) ${ }^{5}$. At the same time, given its fairly high frequency, $\mathrm{CO}[5-4]$ can be observed with reasonably high, subarcsec spatial resolution with both ALMA and IRAM PdBI. We caution, however, that the critical density of $\mathrm{CO}$ [5-4] is an order of magnitude lower than that of HCN (Carilli \& Walter 2013). Strictly speaking, these are not equivalent as tracers. In addition, $\mathrm{CO}[5-4]$ has an excitation temperature of $\sim 83 \mathrm{~K}$, which is much higher than low- $J$ HCN transitions. As such, the low- $J$ lines of these molecules are clearer tracers of density because they do not require the medium to be both warm and dense; we will still need $\mathrm{HCN}[1-0]$ or other dense gas tracers at [1-0] to obtain the total dense gas including the much colder component that will be missed by high- $J \mathrm{CO}$ lines. We do not know, moreover, whether Eq. (4) manifests a direct connection between $\mathrm{CO}[5-4]$ and $L_{\mathrm{IR}}$ or if both $\mathrm{CO}[5-4]$ and $L_{\mathrm{IR}}$ are driven in similar ways by some other parameter, in which case their spatial distribution inside galaxies might differ. Evidence from local galaxies suggests that CO[5-4] emission with $T_{\text {kin }}>50 \mathrm{~K}$, as favored by our LVG modeling, might not be excited by the photodissociation region, but more likely by mechanical heating from shocks (e.g., Rosenberg et al. 2014; Meijerink et al. 2013), which most likely are driven by winds connected to the SF regions. Future comparisons of high spatial resolution maps of $\mathrm{CO}[5-4]$ with $\mathrm{HCN}$, for example, are required to clarify whether this is a viable approach or not, that is, to clarify if $\mathrm{CO}[5-4]$ can be used as a high-fidelity tracer of the star-forming gas.

\subsection{Implications for gas- and dust-scaling laws}

In the previous sections we have presented evidence for the existence of correlations between $\mathrm{CO}$ excitation and $\langle U\rangle$ (Eq. (1)) and between $L_{\mathrm{IR}}$ and $\mathrm{CO}$ [5-4] luminosity, followed by galaxies at $0<z<1.5$, regardless of whether they are MS or starburst systems (we also included some higher- $z$ SB systems, but no $z>2$ MS galaxies). Here we combine these two empirical trends and examine the resulting implications. If the relation between $\mathrm{CO}[5-4] / \mathrm{CO}[1-0]$ and $\langle U\rangle$ were linear, given Eq. (4) and that $\langle U\rangle \propto L_{\mathrm{IR}} / M_{\text {dust }}$, this would imply $L^{\prime} \mathrm{CO}[1-0] / M_{\text {dust }} \sim$ constant. However, we find that the relation is sublinear, with a slope of about $\beta=0.7$, which leads to

$\log \left(L^{\prime} \mathrm{CO}[1-0] / M_{\text {dust }}\right) \propto(1-\beta) \times \log \langle U\rangle$.

This relation supports an accounting for the 3-5 times higher ratio of $L^{\prime} \mathrm{CO}[1-0] / M_{\text {dust }}$ observed for (U)LIRGs compared to local starbursts (Magdis et al. 2012; Tan et al. 2014) given that (U)LIRGs have ten times higher $\langle U\rangle$ than spirals, although the measured difference is not fully accounted for. Given the observed redshift evolution of $\langle U\rangle$ for MS galaxies (Magdis et al. 2012; Béthermin et al. 2015), this relation also predicts a modest rise of the ratio with redshift $\propto(1+z)^{0.4-0.5}$, consistent with the empirical constraint of Tan et al. (2014), who found a trend scaling as $(1+z)^{0.20 \pm 0.27}$.

5 We note, however, that Garcia-Burillo et al. (2012) emphasized that (U)LIRGs have a systematically higher ratio of $L_{\mathrm{IR}}$ to $\mathrm{HCN}$ luminosity by about a factor of two than normal galaxies and suggested a slightly nonlinear correlation between $\mathrm{HCN}$ and $L_{\mathrm{IR}}$. In our case, the comparison of $L^{\prime} \mathrm{CO}[5-4]$ luminosities between $\mathrm{BzK}$ galaxies and local (U)LIRGs has the advantage of being made for very similar $L_{\mathrm{IR}}$ values. 
As discussed by Tan et al. (2014), given the fairly similar functional dependence of both $\alpha_{\mathrm{CO}}$ and the gas-to-dust-mass ratio $(G / D)$ on metallicity for local spirals (see also Sargent et al. 2014; Bolatto et al. 2013; Magdis et al. 2012), one would expect a roughly constant $L^{\prime} \mathrm{CO}[1-0] / M_{\text {dust }}$ ratio for galaxies if metallicity were the only physical parameter determining both $\alpha_{\mathrm{CO}}$ and $G / D$ in galaxies ${ }^{6}$. This does not seem to be the case. If we explicitly exclude the metallicity dependence from both $\alpha_{\mathrm{CO}}$ and $G / D$ assuming they have an identical functional form, $\alpha_{\mathrm{CO}} \propto f(Z) \delta_{\mathrm{CO}}$ and $G / D \propto f(Z) \delta_{G / D}$, we can rewrite Eq. (5) in the form

$\log \left(\delta_{\mathrm{CO}} / \delta_{\mathrm{G} / \mathrm{D}}\right) \propto(\beta-1) \times \log \langle U\rangle$.

This relation reasserts again that $z=0$ (U)LIRGs cannot simultaneously follow the same trend of $\alpha_{\mathrm{CO}}$ and $G / D$ versus metallicity as local spirals. The results of Magdis et al. (2012) suggest that they mainly deviate slightly from the relation of $\alpha_{\mathrm{CO}}$ versus metallicity and follow the $G / D(Z)$ trend reasonably well, but the assessment of metallicity for very dust-embedded systems such as ULIRGs might be biased low (see Tan et al. 2014 for a discussion). Future observations are needed to clarify this question.

More interestingly, this relation has consequences for the evolution of MS galaxies. Given that their $\langle U\rangle$ value increases with redshift, this implies that at $z>0$ they might not simultaneously follow both the same trend of $\alpha_{\mathrm{CO}}$ and $G / D$ versus metallicity as local spiral galaxies. We cannot distinguish the evolution between the $\alpha_{\mathrm{CO}}$ and $G / D$ trend with existing data because inferring gas masses of distant galaxies relies on adopting at least one of these local trends. However, if we were to assume a redshift-invariant $G / D(Z)$ relation, Eq. (6) would imply $\alpha_{\mathrm{CO}}$ values at $z=1.5$ lower by 0.2 dex than those expected if the local scaling of $\alpha_{\mathrm{CO}}$ with metallicity were to also apply to $z=1.5$.

This is similar to the suggestion of Bolatto et al. (2013) that, in addition to the scaling with metallicity, $\alpha_{\mathrm{CO}}$ depends as a secondary parameter on $\left(\Sigma_{\text {Mass }}\right)^{\approx-0.5}$ (their Eq. (31); applicable above a density threshold that is practically always matched by the galaxy populations considered here). Because of the MS, this is equivalent to a dependence on $\left(\Sigma_{\mathrm{SFR}}\right)^{\approx-0.6}$ (using a MS slope of 0.8). Combining our Eqs. (6) and (3) would yield an exponent of -0.73 , not far from the findings of Bolatto and colleagues. In addition, an equivalent scaling with $\Sigma_{\text {SFR }}$ was found by Narayanan et al. (2012) using simulations. Their Eq. (6) would imply an exponent of -0.4 (converting from $\Sigma_{\text {gas }}$ to $\Sigma_{\text {SFR }}$ using a KS relation with a slope of 0.8 ).

\subsection{Massive star-forming clumps as the driver of the high excitation?}

The galaxy BzK-21000 allows us to investigate the possible role played by the giant clumps in driving the high-excitation CO SLEDs of normal MS galaxies at $z=1.5$, as suggested by B15. From inspecting the $\mathrm{CO}$ spectra in the different $J=2,3$, 5 transitions in Fig. 1, we note that this galaxy shows the most obvious variations over the velocity range of the $\mathrm{CO}$ emission. The CO[5-4] emission is much weaker than that of $\mathrm{CO}[2-1]$ in the redshifted part, and most of the recovered $\mathrm{CO}[5-4]$ signal is in the blueshifted part. D10 already showed (see their Fig. 6) that the blue- and redshifted part (relatively to the systemic zerovelocity) corresponds well to the northern and southern portions

\footnotetext{
6 We consider here a fairly high stellar mass regime, $M_{\text {stars }} \gtrsim 10^{10} M_{\odot}$, where galaxies are probably $\mathrm{H}_{2}$ dominated.
}

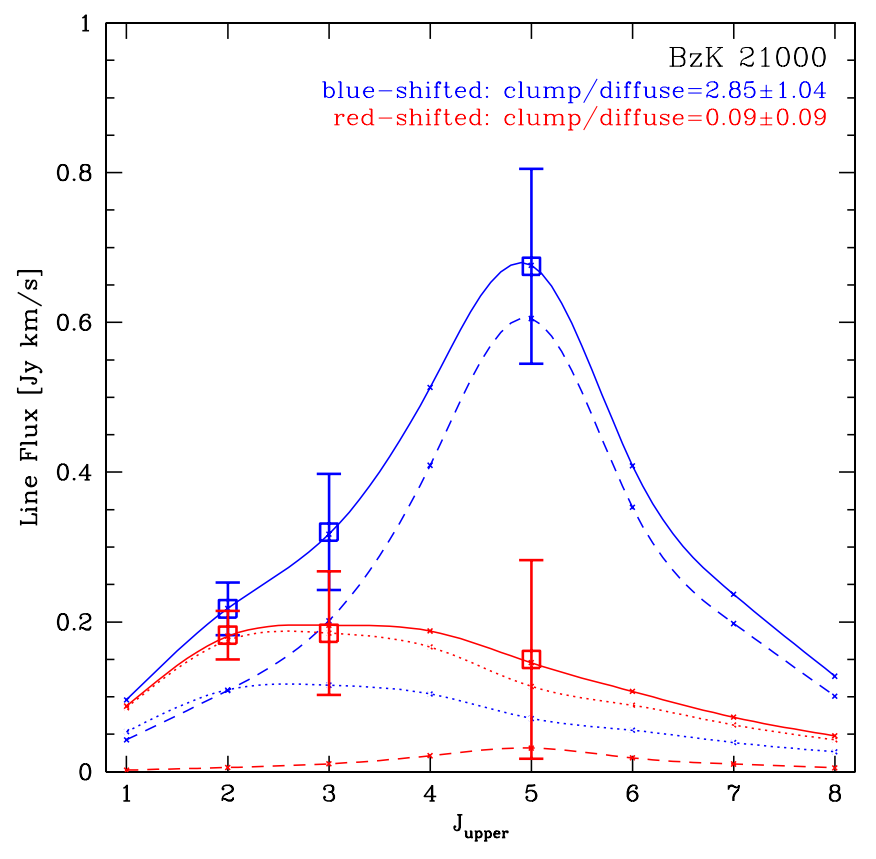

Fig. 14. CO SLED for BzK-21000 shown separately for its blue- and redshifted components (using blue and red points) and decomposed using the clumpy (dashed lines) and diffuse (dotted lines) CO SLEDs from B15 (solid lines are for total SLEDs). The blueshifted component, corresponding to the northern half of the galaxy where most massive clumps are located, is more highly excited, requiring only a modest contribution from the diffuse gas. The redshifted component can only be fitted with diffuse gas. Given the optical and near-IR morphology of BzK-21000, this is consistent with the predictions of the simulations reported by $\mathrm{B} 15$.

of the galaxy, following the global rotation in the disk. We averaged the $\mathrm{CO}$ [3-2] and $\mathrm{CO}$ [5-4] signal over the red and blue velocity ranges separately, as we did for $\mathrm{CO}[2-1]$, and extracted $\mathrm{CO}$ fluxes for all of them by fitting point-like sources at the (fixed) positions of the blue and red components of $\mathrm{CO}[2-1]$ derived from D10, which are offset by $0.8^{\prime \prime}$. We used point sources here because we effectively sample only part of the galaxy each time (so the effective size would be reduced), and also to minimize contamination from the opposite portion of the galaxy. The results of this exercise are shown in the two panels of Fig. 14. We measure a divergence at $\mathrm{CO}[5-4]$ (significant at 2.8 $\sigma$ ) and only a hint of a difference at $\mathrm{CO}$ [3-2].

From Fig. 6 (see also Fig. 5 in D10), it is quite obvious that the northern portion of BzK-21000 is the place where basically all massive clumps are located, in quantitative support of the analysis of B15. We fitted the red and blue CO SLED of BzK-21000 using the clump and diffuse CO SLEDs derived by B15. We find that the redshifted emission can be explained using the diffuse component alone, while the blueshifted emission is dominated by the clumpy component. The normalization of the diffuse component is similar for the blue- and redshifted parts, as expected if such a medium is fairly homogeneously distributed within the galaxy.

Encouraged by the possible correlation between clump location and CO SLED for BzK-21000, which supports the idea of B15 that giant clumps drive the high-excitation component in high- $z$ MS galaxies, we explored the possibility of accounting for excitation variations among our four targets by fitting them with different proportions of clump and diffuse components from B15. The results are shown in Fig. 15. Our average 


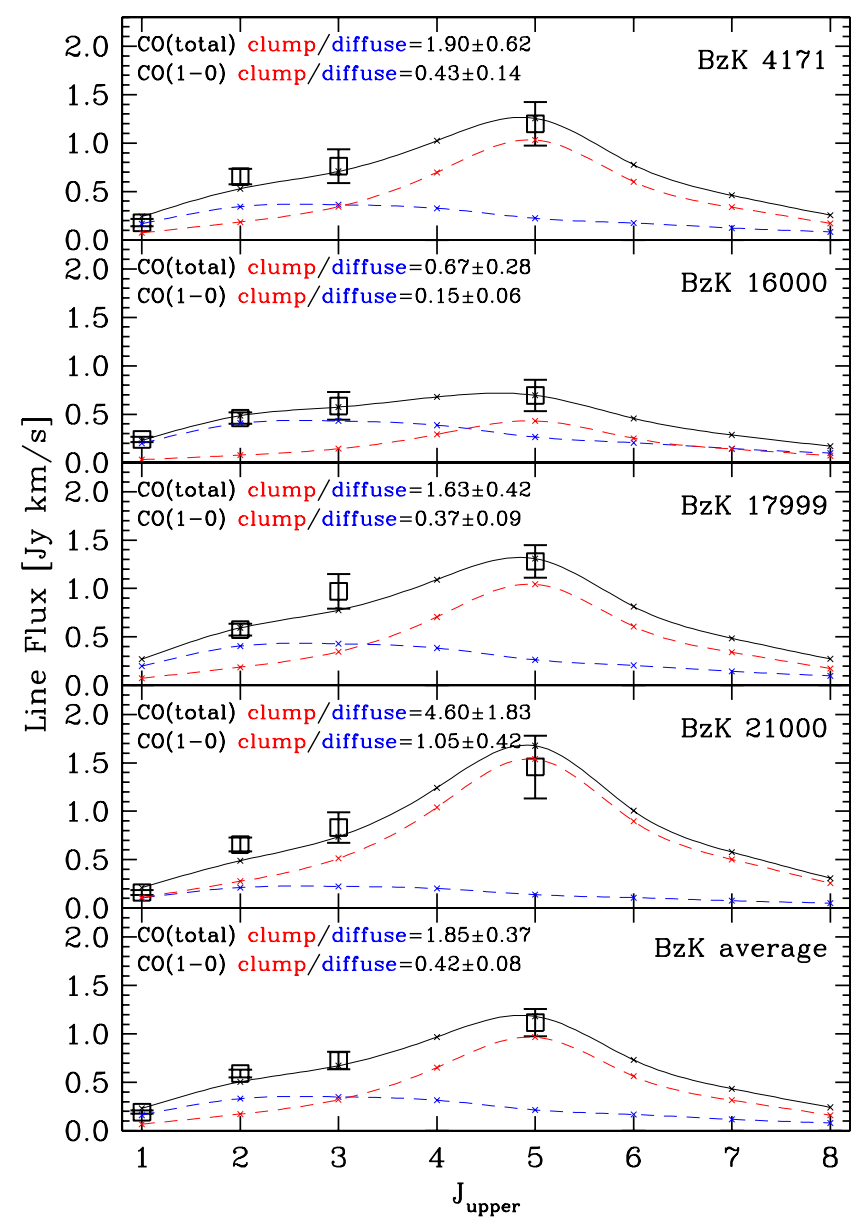

Fig. 15. CO SLEDs of individual BzK galaxies (top four panels) and their average (bottom panel) are fitted using different proportions of the clump (red) and diffuse (blue) CO SLEDs from B15. Results of the fit with their uncertainties are reported as labels in the figure, the $\mathrm{CO}$ (total) ratio is given relative to the high-redshift disk galaxy simulated in B15. For example, for the average $\mathrm{BzK}$ the ratio $\mathrm{CO}$ (total) ratio of $1.85 \mathrm{im}$ plies that we need a clump component 1.85 stronger than that of B15 if the diffuse component is kept fixed.

BzK galaxy requires a larger proportion of clumpy $\mathrm{CO}$ emission, by a factor of 1.8 higher than in the simulations of $\mathrm{B} 15$, probably because they used different gas fractions (see Sect. 4). BzK-21000 requires the strongest clump component and is probably the most clumpy galaxy in our sample, at least in terms of massive clumps (Fig. 6). Instead, BzK-16000 requires a smaller proportion, by a factor 0.67 , of clumpy emission than in B15. This galaxy seems the most evolved in our sample, appearing like a fairly regular large spiral with a massive bulge and lack of very massive clumps. On average, $30 \%$ of the $\mathrm{CO}[1-0]$ emission comes from the clumpy warm component, with values for individual galaxies ranging from $13 \%$ for BzK-16000 to $50 \%$ for BzK-21000.

Figure 16 compares the ratio of $\mathrm{CO}[1-0]$ between clump and diffuse emission with a direct measurement of clumpiness for our four BzK galaxies, measured in the CANDELS HSTWFC3 F160W imaging (Fig. 6). The clumpiness is measured by subtracting a smoothed version from the original images obtained through a boxcar averaging with a kernel size equal to $20 \%$ of the Kron radius - and by evaluating the normalized flux in the pixels with an intensity higher than three times the background standard deviation (see, e.g., Conselice 2003;

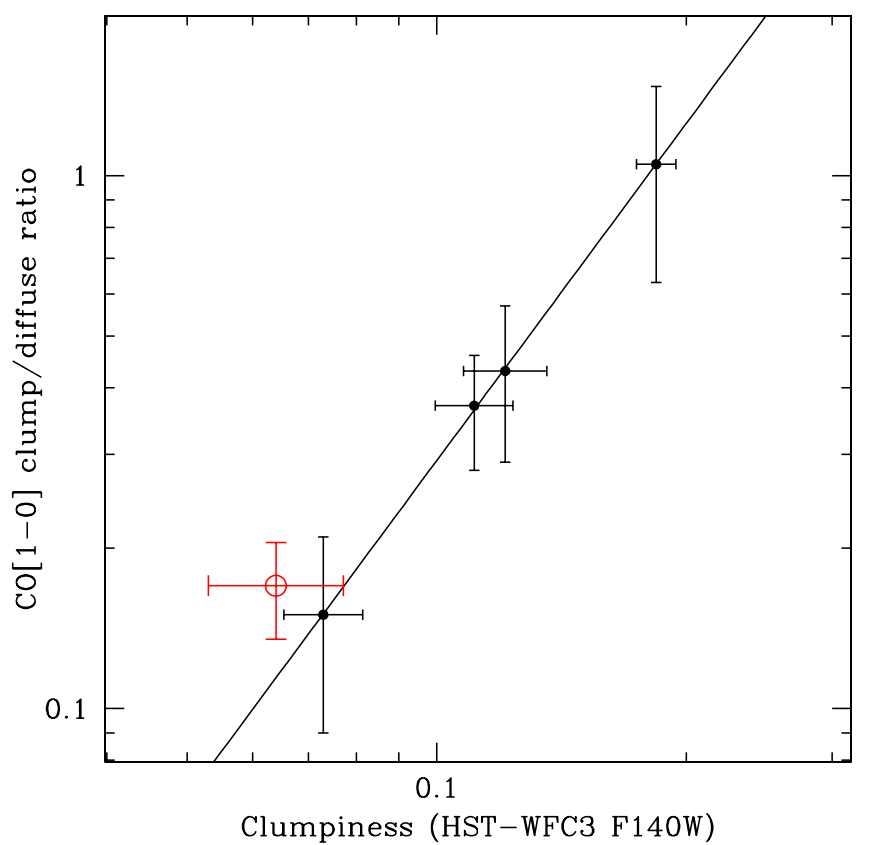

Fig. 16. Ratio of $\mathrm{CO}[1-0]$ emission decomposed between clumpy and diffuse components (based on the CO SLED decomposition, as in Fig. 15) is shown as a function of the clumpiness of the light as seen in the HST-WFC3 F160W imaging (see text for details), corresponding to an optical rest frame of $z=1.5$. The red circle shows the properties of the simulated disk in Bournaud et al. (2014) that appears to fit the trend consistently (solid line). The model of B15 is less clumpy than the average BzK galaxy. The fact that the solid-line fit has a $\chi^{2} \sim 0$ (with 2 degrees of freedom) is just a fortuitous property of the sample, with no further parameter adjustment.

Cibinel et al. 2013). The circular central area (radius $=0.2 \times$ Petrosian radii) was excluded from the calculation to limit the effect of the unresolved inner galaxy region. Errors on the clumpiness were estimated by generating 101 resampled versions of the $F 160 \mathrm{~W}$ images in which each pixel flux was replaced with a random value within the photometric error. The clumpiness was recomputed on each resampled image, and the dispersion over all realizations provides our uncertainty estimate. Although only based on four targets, the correlation between clumpiness and clump and diffuse decomposition of the CO SLED is remarkably good. We emphasize that the measurements of clumpiness and $\mathrm{CO}[1-0]$ decomposition are entirely independent. Good correlations are also found, obviously, between clumpiness and the $\langle U\rangle$ and $\mathrm{CO}[5-4] / \mathrm{CO}[2-1]$ ratios.

It is also tempting to speculate that if the clumps have a much higher $\mathrm{CO}$ excitation than the diffuse component in the rest of the galaxy, it might be expected that in future or putative high spatial resolution Far-IR observations clumps might be found to display higher $\langle U\rangle$ values than the rest of the galaxies (i.e., warmer Far-IR SEDs), which might be related to a higher SFE and/or lower metallicities. Both options appear viable at this stage, with a lower gas-phase metallicity being plausible if they form from collapse of at least partially newly accreted or pristine gas. Evidence for higher SFEs in the clumps at least in their collapse phases were presented in Zanella et al. (2015). Verifying this possibility will require the combination of multi-wavelength and high-resolution tracers of the dust continuum emission, spanning from relatively short wavelengths at rest frame $<100 \mu \mathrm{m}$ all the way to the Rayleigh-Jeans tail at $500 \mu \mathrm{m}$ rest frame or longer. This should be within the capabilities of 


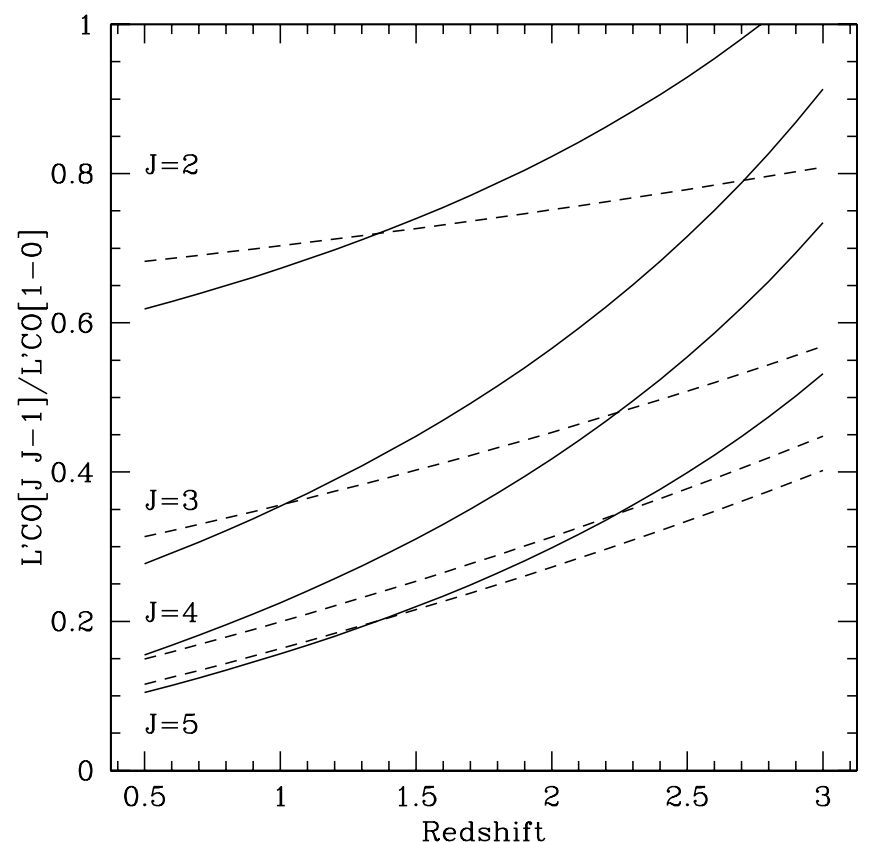

Fig. 17. Predicted average luminosity ratios for MS galaxies of various $\mathrm{CO}$ transitions normalized to $L^{\prime} \mathrm{CO}[1-0]$, exploiting the correlation between CO SLED excitation and $\langle U\rangle$ and between $\langle U\rangle$ and redshift (Magdis et al. 2012; Béthermin et al. 2015). This is computed for the $J=2,3,4,5$ transitions, curves from top to bottom. We considered two-component cold and warm CO SLEDs, corresponding to the different solutions shown in Fig. 10, with the warmer component peaking at $J=5$ (solid) or 7 (dashed).

ALMA for $z \gtrsim 2$ galaxies, especially if band 10 observations are eventually developed.

\subsection{Predicting the evolving CO SLED of MS galaxies versus redshift}

The correlation of $\mathrm{CO}[5-4] / \mathrm{CO}[2-1]$ with $\langle U\rangle$ can be used to compute expectations for the excitation of normal MS SF galaxies as a function of redshift. The rise of $U \propto(1+z)^{1.2}$ (Magdis et al. 2012) or possibly steeper (Béthermin et al. 2015) suggests that typical SF galaxies at intermediate redshifts $z \sim 0.5-1$ will be on average less excited than those at $z=1.5$, perhaps with CO SLEDs more similar to that of BzK-16000. On the other hand, it appears that $\langle U\rangle$ continues to grow steeply all the way to at least $z \sim 4$ (Béthermin et al. 2015), suggesting that typical SF galaxies will increasingly excited in their $\mathrm{CO}$ emission toward higher redshifts. By $z \sim 3$ their intensity of radiation field might become similar to that of local (U)LIRGs, and similar CO SLEDs might be expected at that point, in large part probably driven by the decline of metallicity toward high- $z$ (while we expect this to be primarily a SFE effect in (U)LIRGs).

Following this idea, we have attempted calculations of expected CO SLED versus redshift for MS galaxies. We assumed that the SLED can be decomposed into a cold and a warm component, and varied their relative strength to reproduce the trend in Eq. (1) and Fig. 12. We chose the functional form developed in Béthermin et al. (2015) of the $\langle U\rangle(z)$ relation, which scales like $(1+z)^{1.8}$. In Fig. 17 we plot the resulting luminosity ratios for a warm component peaking at $J \sim 5$ (solid lines; Fig. 10) and $J \sim 7$ (dashed lines), based on the LVG modeling (Sect. 5). These tracks can be used to correct $\mathrm{CO}$ luminosities of normal
MS galaxies in $J \leq 5$ transitions to $L^{\prime} \mathrm{CO}[1-0]$. When these corrections are applied to individual galaxies, one should consider that in addition to the uncertainties related to the choice of the underlying warm or cold components, the dispersion in the $\langle U\rangle(z)$ relation (of about 0.2 dex, see Magdis et al. 2012) and the intrinsic dispersion in the $\langle U\rangle$ versus $\mathrm{CO}[5-4] / \mathrm{CO}[2-1]$ trend (0.11 dex in our estimate) imply a scatter of at least 0.15 dex for individual expectations of the $\mathrm{CO}[5-4] / \mathrm{CO}[2-1]$ ratio.

\section{Summary and conclusions}

We have obtained CO SLEDs of four normal galaxies at $z=1.5$ including measurements in the $\mathrm{CO}[1-0], \mathrm{CO}[2-1]$, $\mathrm{CO}[3-2]$, and $\mathrm{CO}[5-4]$ transitions. All $\mathrm{CO}$ [5-4] observations and $\mathrm{CO}[3-2]$ observations of three galaxies were presented here for the first time, while the rest of the observations were taken from D10, Dannerbauer et al. (2009), and Aravena et al. (2010, 2014). This is the first time that such detailed SLEDs are available for normal unlensed galaxies, which were selected in the near-IR (not by means of their very high $L_{\mathrm{IR}}$ ) and belong to the star-forming main sequence at their redshift. The measurements are thus representative of typical disk-like galaxies at high redshifts, which are the cause for the bulk of the cosmic SFRD. The main results can be summarized as follows:

- While the CO SLEDs of all four galaxies are consistent with a Milky-Way-like, low excitation up to $\mathrm{CO}$ [3-2] (confirming the results of Dannerbauer et al. 2009; Aravena et al. 2010, 2014), we detect substantially excited CO[5-4] emission well in excess of that in local spirals in our sample. The CO SLEDs of $z=1.5$ disks evolve substantially with respect to their local main-sequence counterparts when considering $J>3$ transitions. However, the CO SLEDs of our sample $z=1.5$ galaxies are less excited than those of local (U)LIRGs and than the average SMGs, displaying weaker ratios of $\mathrm{CO}[5-4]$ to low- $J \mathrm{CO}$.

- Existing predictions for the SLEDs of this population correctly reproduce the enhancement of $\mathrm{CO}[5-4]$ emission with respect to local galaxies. However, in detail, P12 predicted too highly excited emission, B15 predicted less highly excited emission, while Narayanan \& Krumholz (2014) predicted a shape that rises too strongly over the $\mathrm{CO}[3-2]-$ $\mathrm{CO}[4-3]$ transitions. Variations observed within individual galaxies in our sample approach values at either of the extremes of the models.

- We presented LVG modeling of the SLEDs of the $z=1.5$ galaxies. At least two components are required to reproduce the SLEDs, with the most relevant constraints applying to their densities. A $n<10^{3} \mathrm{~cm}^{-3}$ low-excitation component is required, together with a second component at higher density $n>10^{3.5} \mathrm{~cm}^{-3}$ with possibly higher temperatures.

- By comparing different galaxy populations including MS and SB galaxies at low and high redshifts, we found that a good tracer of the overall $\mathrm{CO}$ excitation, as parametrized by the $\mathrm{CO}[5-4] / \mathrm{CO}[2-1]$ ratio, is the intensity of the radiation field $\langle U\rangle$, which is related to the effective dust temperature. We found a sublinear correlation with $\langle U\rangle$, having a logarithmic slope of 0.6. The correlation with $\langle U\rangle$ allowed us to predict that intermediate-redshift SF galaxies (e.g., at $z \sim 0.5-1)$ will have an excitation intermediate between local spirals and BzK galaxies, while typical $z>3 \mathrm{SF}$ galaxies might have CO SLEDs with excitations similar to those of local (U)LIRGs. This could counterbalance the lower CO luminosities expected at high redshift because of the higher 
$\alpha_{\mathrm{CO}}$ driven by the declining metallicity, by making higher- $J$ transitions more easily observable.

- We found that the $z=1.5$ normal galaxies follow, within the uncertainties, the same linear correlation between $\mathrm{CO}$ [5-4] luminosity and $L_{\mathrm{IR}}$ as local spirals, (U)LIRGs, and SMGs. The CO[5-4] emission appears to be related to the starforming dense gas, regardless of the nature (main sequence vs. starburst) of the galaxy. This is different from what is observed for low- $J$ CO emission. Future observations will clarify whether $\mathrm{CO}[5-4]$ can be used as a more effective (i.e., less time-consuming) alternative to $\mathrm{HCN}$ to study the dense SF gas in galaxies.

- Within our sample, we found evidence supporting the idea that the high-excitation $\mathrm{CO}$ emission in the BzK galaxies at $z=1.5$ originates from their massive clumps, in agreement with the predictions of $\mathrm{B} 15$.

To complete the observations of $\mathrm{CO}[3-2]$ and $\mathrm{CO}[5-4]$ presented in this paper, we required a total on-source time of $82 \mathrm{~h}$ with the IRAM PdBI, while $62 \mathrm{~h}$ were used by D10 to secure high $\mathrm{S} / \mathrm{N}$ observations of $\mathrm{CO}[2-1]$. Overall, counting standard overheads, this corresponds to a total of about $230 \mathrm{~h}$ of telescope time at PdBI for three $\mathrm{CO}$ transitions over four galaxies. ALMA can now complete such observations more than a hundred times faster, and we hope that the results presented here will be useful to guide the exploration of much larger samples with ALMA. Nevertheless, before ALMA starts observing below $45 \mathrm{GHz}$, obtaining $\mathrm{CO}[1-0]$ flux measurements for $z>1$ galaxies at the VLA will still be a very time-consuming endeavour for years to come: over $250 \mathrm{~h}$ were used to secure the highquality $\mathrm{CO}[1-0]$ measurements for the three galaxies we used here. However, our results suggest that in most cases, $\mathrm{CO}[2-1]$ can be a fair substitute because this transition is on average only marginally subthermally excited. This is of course progressively less the case for higher- $J$ transitions, starting from $\mathrm{CO}[3-2]$ and especially for $\mathrm{CO}[5-4]$, which is seen to be more and more directly sensitive to the SFR and not to the total $\mathrm{H}_{2}$ gas reservoir.

Acknowledgements. We thank Ronin Wu and Aurélie Remy-Ruyer for providing information on M 83 and IC 342. We are grateful to Desika Narayanan and Padelis Papadopoulos for discussions and to the anonymous referee for a constructive report. This work was based on observations carried out with the IRAM PdBI, supported by INSU/CNRS (France), MPG (Germany), and IGN (Spain). The National Radio Astronomy Observatory is a facility of the National Science Foundation operated under cooperative agreement by Associated Universities, Inc. We acknowledge the use of GILDAS software (http://www.iram.fr/ IRAMFR/GILDAS). We acknowledge the support of the ERC-StG UPGAL 240039 and ANR-08-JCJC-0008 grants.

\section{References}

Aravena, M., Carilli, C., Daddi, E., et al. 2010, ApJ, 718, 177 Aravena, M., Hodge, J. A., Wagg, J., et al. 2014, MNRAS, 442, 558 Béthermin, M., Daddi, E., Magdis, G., et al. 2015, A\&A, 573, A113 Bothwell, M. S., Smail, I., Chapman, S. C., et al. 2013, MNRAS, 429, 3047 Bournaud F., Daddi, E., Elmegreen, B. G., et al. 2008, A\&A, 486, 741 Bournaud, F., Dekel, A., Teyssier, R., et al. 2011, ApJ, 741, L33 Bournaud, F., Juneau, S., Le Floc'h, E., et al. 2012, ApJ, 757, 81 Bournaud, F., Daddi, E., Weiss, A., et al. 2015, A\&A, 575, A56 Burgarella, D., Buat, V., Gruppioni, C., et al. 2013, A\&A, 554, A70 Carilli, C. L., \& Walter, F. 2013, ARA\&A, 51, 105

Carilli, C. L., Daddi, E., Riechers, D., et al. 2010, ApJ, 714, 1407 Chabrier, G. 2003, PASP, 115, 763

Chanial, P., Flores, H., Guiderdoni, B., et al. 2007, A\&A, 462, 81 Cibinel, A., Carollo, C. M., Lilly, S. J., et al. 2013, ApJ, 776, 72 Combes, F., Rex, M., Rawle, T. D., et al. 2012, A\&A, 538, L4 Conselice, C. J. 2003, ApJS, 147, 1

Cox, P., Krips, M., Neri, R., et al. 2011, ApJ, 740, 63 da Cunha, E., Charmandaris, V., Díaz-Santos, T., et al. 2010, A\&A, 523, A78 Daddi, E., Dickinson, M., Morrison, G., et al. 2007, ApJ, 670, 156 (D07) Daddi, E., Dannerbauer, H., Elbaz, D., et al. 2008, ApJ, 673, L21

Daddi, E., Dannerbauer, H., Stern, D., et al. 2009a, ApJ, 694, 1517

Daddi, E., Dannerbauer, H., Krips, M., et al. 2009b, ApJ, 695, L176

Daddi, E., Bournaud, F., Walter, F., et al. 2010a, ApJ, 713, 686 (D10)

Daddi, E., Elbaz, D., Walter, F., et al. 2010b, ApJ, 714, L118

Dale, D. A., Aniano, G., Engelbracht, C. W., et al. 2012, ApJ, 745, 95

Danielson, A. L. R., Swinbank, A. M., Smail, I., et al. 2011, MNRAS, 410, 1687

Dannerbauer, H., Daddi, E., Riechers, D. A., et al. 2009, ApJ, 698, L178

Downes, D., \& Solomon, P. M. 1998, ApJ, 507, 615

Draine, B. T., \& Li, A. 2007, ApJ, 657, 810

Elbaz, D., Daddi, E., Le Borgne, D., et al. 2007, A\&A, 468, 33

Elbaz, D., Dickinson, M., Hwang, H. S., et al. 2011, A\&A, 533, A119

Erb, D. K., Shapley, A. E., Pettini, M., et al. 2006, ApJ, 644, 813

Flower, D. R. 2001, J. Phys. B: At. Mol. Phys., 34, 2731

Fixsen D. J., Bennett C. L., \& Mather J. C., 1999, ApJ, 526, 207

Förster Schreiber, N. M., Genzel, R., Bouché, N., et al. 2009, ApJ, 706, 1364

Gao, Y., \& Solomon, P. M. 2004a, ApJ, 606, 271

Gao, Y., \& Solomon, P. M. 2004b, ApJS, 152, 63

Gao, Y., Carilli, C. L., Solomon, P. M., \& Vanden Bout, P. A. 2007, ApJ, 660, L93

García-Burillo, S., Usero, A., Alonso-Herrero, A., et al. 2012, A\&A, 539, A8

Geach, J. E., Smail, I., Moran, S. M., et al. 2011, ApJ, 730, L19

Genzel R., Tacconi, L. J., Eisenhauer, F., et al. 2006, Nature, 442, 786

Genzel, R., Tacconi, L. J., Gracia-Carpio, J., et al. 2010, MNRAS, 407, 2091

Genzel, R., Tacconi, L. J., Lutz, D., et al. 2015, ApJ, 800, 20

Goldreich, P., \& Kwan, J. 1974, ApJ, 189, 441

Greve, T. R., Leonidaki, I., Xilouris, E. M., et al. 2014, ApJ, 794, 142

Grogin N. A., Kocevski, D. D., Faber, S. M., et al. 2011, ApJS, 197, 35

Hodge, J. A., Carilli, C. L., Walter, F., et al. 2012, ApJ, 760, 11

Hodge, J. A., Carilli, C. L., Walter, F., Daddi, E., \& Riechers, D. 2013, ApJ, 776, 22

Hodge, J A., Riechers, D., Decarli, R., et al. 2015, ApJ, 798, L18

Hopkins, P. F., \& Quataert, E. 2011, MNRAS, 415, 1027

Hwang, H. S., Elbaz, D., Magdis, G., et al. 2010, MNRAS, 409, 75

Ivison, R. J., Papadopoulos, P. P., Smail, I., et al. 2011, MNRAS, 412, 1913

Karim, A., Schinnerer, E., Martínez-Sansigre, A., et al. 2011, ApJ, 730, 61

Kashino, D., Silverman, J. D., Rodighiero, G., et al. 2013, ApJ, 777, L8

Kennicutt, R. C., Jr. 1998, ApJ, 498, 541

Koekemoer, A. M., Faber, S. M., Ferguson, H. C., et al. 2011, ApJS, 197, 36

Lagos, C. del P., Bayet, E., Baugh, C. M., et al. 2012, MNRAS, 426, 2142

Leroy, A. K., Walter, F., Brinks, E., et al. 2008, AJ, 136, 2782

Leroy, A. K., Walter, F., Bigiel, F., et al. 2009, AJ, 137, 4670

Lu, N., Zhao, Y., Xu, C. K., et al. 2014, ApJ, 787, L23

Madau, P., \& Dickinson, M., 2014, ARA\&A, 52, 415

Magdis, G. E., Elbaz, D., Daddi, E., et al. 2010, ApJ, 714, 1740

Magdis, G. E., Daddi, E., Elbaz, D., et al. 2011, ApJ, 740, L15

Magdis, G. E., Daddi, E., Sargent, M., et al. 2012, ApJ, 758, L9

Magnelli, B., Popesso, P., Berta, S., et al. 2013, A\&A, 553, A132

Maier, C., Lilly, S. J., Ziegler, B. L., et al. 2014, ApJ, 792, 3

Meijerink, R., Kristensen, L. E., Weiß, A., et al. 2013, ApJ, 762, L16

Narayanan, D., \& Krumholz, M. R. 2014, MNRAS, 442, 1411

Narayanan D., Krumholz M. R., Ostriker E. C., \& Hernquist L., 2012, MNRAS, 421,3127

Noeske, K. G., Weiner, B. J., Faber, S. M., et al. 2007, ApJ, 660, L43

Pannella, M., Carilli, C. L., Daddi, E., et al. 2009, ApJ, 698, L116

Pannella, M., Elbaz, D., Daddi, E., et al. 2014, ApJ, submitted [arXiv: 1407.5072]

Papadopoulos, P. P., van der Werf, P. P., Xilouris, E. M., et al. 2012, MNRAS, 426, 2601 (P12)

Papadopoulos P. P., Zhang, Z.-Y., Xilouris, E. M., et al. 2014, ApJ, 788, 153

Peng, C. Y., Ho, L. C., Impey, C. D., \& Rix, H.-W. 2010, AJ, 139, 2097

Price, S. H., Kriek, M., Brammer, G. B., et al. 2014, ApJ, 788, 86

Pope, A., Wagg, J., Frayer, D., et al. 2013, ApJ, 772, 92

Popping G., Pérez-Beaupuits, J. P., Spaans, M., Trager, S. C., \& Somerville, R. S. 2014, MNRAS, 444, 1301

Riechers, D. A., Hodge, J., Walter, F., Carilli, C. L., \& Bertoldi, F. 2011, ApJ, 739, L31

Riechers, D. A., Bradford, C. M., Clements, D. L., et al. 2013, Nature, 496, 329

Rigopoulou, D., Hurley, P. D., Swinyard, B. M., et al. 2013, MNRAS, 434, 2051

Rodighiero, G., Daddi, E., Baronchelli, I., et al. 2011, ApJ, 739, L40

Rodighiero, G., Renzini, A., Daddi, E., et al. 2014, MNRAS, 443, 19

Rosenberg, M. J. F., Kazandjian, M. V., van der Werf, P. P., et al. 2014, A\&A, 564, A 126

Rujopakarn, W., Rieke, G. H., Eisenstein, D. J., \& Juneau, S. 2011, ApJ, 726, 93

Salmi, F., Daddi, E., Elbaz, D., et al. 2012, ApJ, 754, L14

Santini, P., Maiolino, R., Magnelli, B., et al. 2014, A\&A, 562, A30 
Sargent, M. T., Béthermin, M., Daddi, E., \& Elbaz, D. 2012, ApJ, 747, L31 Sargent, M. T., Daddi, E., Béthermin, M., et al. 2014, ApJ, 793, 19 Schreiber C., Pannella, M., Elbaz D., et al. 2015, A\&A, 575, A74

Scott, K. S., Lupu, R. E., Aguirre, J. E., et al. 2011, ApJ, 733, 29 Scoville, N. Z., 1994, ASP Conf. Ser., 59, 312

Scoville, N. Z., \& Solomon, P. M. 1974, ApJ, 187, L67

Scoville, N., Aussel, H., Sheth, K., et al. 2014, ApJ, 783, 84

Solomon, P. M., \& Vanden Bout, P. A. 2005, ARA\&A, 43, 677

Spilker, J. S., Marrone, D. P., Aguirre, J. E., et al. 2014, ApJ, 785, 149

Steidel, C. C., Rudie, G. C., Strom, A. L., et al. 2014, ApJ, 795, 165

Tacconi, L. J., Genzel, R., Neri, R., et al. 2010, Nature, 463, 781

Tacconi, L. J., Neri, R., Genzel, R., et al. 2013, ApJ, 768, 74

Tan, Q., Daddi, E., Sargent, M., et al. 2013, ApJ, 776, L24

Tan, Q., Daddi, E., Magdis, G., et al. 2014, A\&A, 569, A88
Troncoso, P., Maiolino, R., Sommariva, V., et al. 2014, A\&A, 563, A58

Valentino, F., Daddi, E., Strazzullo, V., et al. 2015, ApJ, 801, 132

van der Wel, A., Franx, M., van Dokkum, P. G., et al. 2014, ApJ, 788, 28

Weiss, A., Downes, D., Walter, F., \& Henkel, C. 2007, in From Z-Machines to ALMA: (Sub)Millimeter Spectroscopy of Galaxies, ASP Conf. Ser., 375, 25 Whitaker, K. E., van Dokkum, P. G., Brammer, G., \& Franx, M. 2012, ApJ, 754, L29

Whitaker K. E., Franx, M., Leja, J., et al. 2014, ApJ, 795, 104

Wu, R., Madden S., Galliano, F., et al. 2015, A\&A, 575, A88

Wuyts, S., Förster Schreiber, N. M., van der Wel, A., et al. 2011, ApJ, 742, 96

Zahid, H. J., Kashino, D., Silverman, J. D., et al. 2014, ApJ, 792, 75

Zanella, A., Daddi, E., Le Floc'h, E., et al. 2015, Nature, in press

Zhang Z.-Y., Gao Y., Henkel C., et al. 2014, ApJ, 784, L31 